ORGANIC LETTERS

SUPPORTING INFORMATION

\title{
An Approach to Pancratistatins via Ring-Closing Metathesis: Efficient Synthesis of Novel 1-Aryl-1-deoxyconduritols F
}

\author{
Oleg N. Nadein and Alexander Kornienko* \\ Department of Chemistry, New Mexico Institute of Mining and Technology, \\ Socorro, New Mexico 87801
}

\section{EXPERIMENTAL SECTION}

All commercially obtained reagents were used without purification unless otherwise noted. THF was distilled from sodium-benzophenone ketyl prior to use. Dichloromethane and methanol were distilled from calcium chloride. Reactions were monitored by TLC (EM Science, Silica Gel $60 \mathrm{~F}_{254}$, $250 \mu \mathrm{m})$ and visualized with UV light and ceric ammonium molybdate solution. Flash chromatography was performed on silica gel (AdTech Flash Silica, 32-63 $\mu \mathrm{m}, 60 \AA$ pore size). ${ }^{1} \mathrm{H}$ and ${ }^{13} \mathrm{C}$ NMR spectra were recorded on JEOL $300 \mathrm{MHz}$ spectrometer. The structures of all new compounds were confirmed by double resonance and hetero-correlation experiments. HRMS was performed at UCLA mass spectrometry facility.<smiles>CO[C@H]1OC[C@@H](O)[C@H](O)[C@H]1O</smiles>

Mixture of $\alpha$ - and $\beta$-methyl $\mathbf{D}$-xylopyranosides. Methanolic solution of $1 \% \mathrm{HCl}$ was prepared by a careful addition of $\mathrm{SOCl}_{2}(5 \mathrm{~mL}, 0.069 \mathrm{~mol})$ to stirred dry $\mathrm{MeOH}(250 \mathrm{~mL})$ at $0{ }^{\circ} \mathrm{C}$. D-xylose $(25$ $\mathrm{g}, 0.17 \mathrm{~mol}$ ) was added in one portion and the resulting solution was refluxed for $4 \mathrm{~h}$. The reaction mixture was allowed to cool to rt, neutralized by the addition of solid $\mathrm{NaHCO}_{3}(17.5 \mathrm{~g}, 0.21 \mathrm{~mol})$ and concentrated under reduced pressure. The residue was dissolved in $\mathrm{EtOH}(200 \mathrm{~mL})$, the solution was concentrated to one half of the original volume, toluene $(100 \mathrm{~mL})$ was added and the mixture was concentrated to dryness. The residual viscous oil was used without purification in the next step.<smiles>CO[C@H]1OC[C@@H](O[GaH2])[C@H](OCc2ccccc2)[C@H]1Cc1ccccc1</smiles>

Mixture of $\boldsymbol{\alpha}$ - and $\boldsymbol{\beta}$-methyl 2,3,4-tri- $\boldsymbol{O}$-benzyl-D-xylopyranosides. To $\mathrm{NaH} 60 \%$ suspension in mineral oil $(34 \mathrm{~g}, 0.85 \mathrm{~mol})$ in a $1 \mathrm{~L}$ flask was added a solution of methyl xylosides from the previous step in DMF $(500 \mathrm{~mL})$ in $100 \mathrm{~mL}$ portions at $0{ }^{\circ} \mathrm{C}$ with vigorous stirring. After the hydrogen evolution was complete the mixture was treated with $\mathrm{Bu}_{4} \mathrm{NI}(11 \mathrm{~g}, 0.03 \mathrm{~mol})$ and $\mathrm{BnCl}$ 
(70 mL, $0.61 \mathrm{~mol}$ ) and stirred for $30 \mathrm{~min}$ at $0{ }^{\circ} \mathrm{C}$ and then overnight at $\mathrm{rt}$. The reaction mixture was carefully quenched with cold aqueous solution of $10 \% \mathrm{NH}_{4} \mathrm{Cl}(130 \mathrm{~g})$. Water $(400 \mathrm{~mL})$ was added to the mixture and the aqueous layer was extracted with ether $(3 \times 200 \mathrm{~mL})$. The combined organic extracts were washed with water $(2 \times 300 \mathrm{~mL})$, then dried $\left(\mathrm{MgSO}_{4}\right)$, and concentrated under reduced pressure. The residual oil (about $250 \mathrm{~mL}$ ) was subjected to the next step without purification.

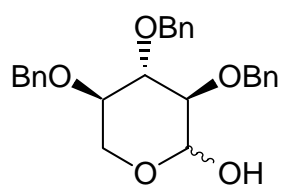

2,3,4-Tri- $\boldsymbol{O}$-benzyl-D-xylopyranose. ${ }^{1,2}$ The crude mixture of xylosides from the previous step was heated under reflux for $10 \mathrm{~h}$ with $1 \mathrm{M} \mathrm{H}_{2} \mathrm{SO}_{4}(210 \mathrm{~mL})$ - AcOH $(240 \mathrm{~mL})$ - dioxane $(220 \mathrm{~mL})$. After allowing the mixture to cool to rt, hexane $(100 \mathrm{~mL})$ and water $(800 \mathrm{~mL})$ were added with intense stirring. The separated precipitate was collected by filtration, washed with hexane $(2 \times 250$ $\mathrm{mL}$ ) and air-dried. Recrystallization from methanol gave 2,3,4-tri- $O$-benzyl-D-xylose as white needles (50.7 g, $71 \%)$; mp $137-138^{\circ} \mathrm{C}\left(139-142{ }^{\circ} \mathrm{C}\right.$, lit. $\left.{ }^{2}\right) ; R_{f} 0.45$ (33\% EtOAc/hexanes).<smiles>C=CC(OCC(Br)(Br)c1ccccc1)[C@@H](O)C(O)CO</smiles>

(2R,3R,4S)-2,3,4-Tri(benzyloxy)-5-hexene-1-ol. ${ }^{3,4}$ To a stirred suspension of methyltriphenylphosphonium bromide $(42 \mathrm{~g}, 0.1 \mathrm{~mol})$ in THF $(150 \mathrm{~mL})$ was added BuLi $(50 \mathrm{~mL}$ of $2 \mathrm{M}$ solution in pentane, $0.1 \mathrm{~mol}$ ) dropwise at $0{ }^{\circ} \mathrm{C}$. The mixture was stirred for $2 \mathrm{~h}$ at $\mathrm{rt}$. To the above red solution was added tri- $O$-benzylxylopyranose $(20 \mathrm{~g}, 0.048 \mathrm{~mol})$ in THF $(150 \mathrm{~mL})$ dropwise at $0{ }^{\circ} \mathrm{C}$. The reaction mixture was stirred overnight at $\mathrm{rt}$ and then at reflux for $2 \mathrm{~h} .1 \mathrm{M} \mathrm{NH}_{4} \mathrm{Cl}(200 \mathrm{~mL})$ was added at $\mathrm{rt}$ to the reaction mixture and the latter was extracted with ether $(3 \times 200 \mathrm{~mL})$. The organic extracts were combined, washed with brine and dried $\left(\mathrm{MgSO}_{4}\right)$. After the removal of the solvent, the residue was passed through a short column of silica gel with gradients: $15 \%, 35 \% \mathrm{EtOAc/hexanes,}$ to obtain $16 \mathrm{~g}(80 \%)$ of the enol product; $R_{f} 0.38$ (33\% EtOAc/hexanes).<smiles>C=CC(OCC)C(Br)c1ccccc1</smiles>

Methyl (2E,4R,5R,6S)-4,5,6-tri(benzyloxy)-2,7-octadienoate (6). ${ }^{4}$ To oxalyl chloride (27 $\mathrm{mL}$ of $2 \mathrm{M}$ in $\left.\mathrm{CH}_{2} \mathrm{Cl}_{2}, 54 \mathrm{mmol}\right)$ in dry $\mathrm{CH}_{2} \mathrm{Cl}_{2}(100 \mathrm{~mL})$ at $-78{ }^{\circ} \mathrm{C}$ was added DMSO $(7.8 \mathrm{~mL}, 110 \mathrm{mmol})$ in $\mathrm{CH}_{2} \mathrm{Cl}_{2}(40 \mathrm{~mL})$ over $20 \mathrm{~min}$ and the mixture was stirred for $40 \mathrm{~min}$. The above alcohol $(10 \mathrm{~g}, 24$ mmol) in $\mathrm{CH}_{2} \mathrm{Cl}_{2}(40 \mathrm{~mL})$ was added over $30 \mathrm{~min}$ and the mixture was stirred for an additional $1 \mathrm{~h}$. Triethylamine $(20 \mathrm{~mL}, 133 \mathrm{mmol})$ in $\mathrm{CH}_{2} \mathrm{Cl}_{2}(40 \mathrm{~mL})$ was added over 20 min and the white slurry was stirred for $30 \mathrm{~min}$ at $-78{ }^{\circ} \mathrm{C}$. To the cold reaction mixture was added methyl (triphenylphosphoranylidene)acetate $(18 \mathrm{~g}, 54 \mathrm{mmol})$ in one portion, and the resulting mixture was stirred for $10 \mathrm{~h}$ while it was allowed to warm up to $\mathrm{rt}$. Water $(300 \mathrm{~mL})$ was added to the reaction mixture, the two layers were separated and the aqueous layer was extracted with $\mathrm{CH}_{2} \mathrm{Cl}_{2}(3 \times 200 \mathrm{~mL})$. The combined 
organic layers were washed with brine, dried $\left(\mathrm{MgSO}_{4}\right)$ and evaporated under reduced pressure. The residual oil was split in two fractions, which were presorbed on silica gel and purified by chromatography with gradients: $5 \%, 15 \% \mathrm{EtOAc/hexanes}$ to afford enoate $6(9.7 \mathrm{~g}, 85 \%)$ as a colorless oil; $R_{f} 0.55$ ( $25 \%$ EtOAc/hexanes).

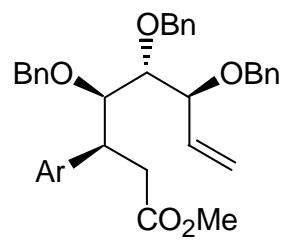

\section{General procedure for aryl cuprate addition.}

$3 \mathrm{~mL}$ of the required arylbromide ${ }^{5}(30 \mathrm{mmol})$ solution in THF $(60 \mathrm{~mL})$ was added to magnesium turnings $(0.79 \mathrm{~g}, 33 \mathrm{mmol})$ in THF $(10 \mathrm{~mL})$. Magnesium was crushed in the flask with a glass rod until the solution started to turn yellowish and warm. The rest of aryl bromide solution was added dropwise to keep gentle boiling of the stirred reaction mixture. After it was cooled to rt, the solution of a Grignard reagent was transferred to a slurry of $\mathrm{CuI}(2.86 \mathrm{~g}, 15 \mathrm{mmol})$ in THF $(15 \mathrm{~mL})$ at $0{ }^{\circ} \mathrm{C}$ via cannula. The resulting mixture was stirred at $0{ }^{\circ} \mathrm{C}$ for $20 \mathrm{~min}$, then treated with $\mathrm{TMSCl}(3.58 \mathrm{~g}$, $33 \mathrm{mmol})$ at $-78^{\circ} \mathrm{C}$, followed by the addition of enoate $6(1.42 \mathrm{~g}, 3 \mathrm{mmol})$ in THF $(15 \mathrm{~mL})$. Stirring was continued overnight, while the mixture was allowed to warm up to rt. The reaction mixture was quenched with a mixture of conc. $\mathrm{NH}_{4} \mathrm{OH}-$ sat. $\mathrm{NH}_{4} \mathrm{Cl}(1: 9,100 \mathrm{~mL})$, diluted with ether and separated. The aqueous layer was extracted with ether $(3 \times 100 \mathrm{~mL})$ and the combined organic layers were washed with brine, dried $\left(\mathrm{MgSO}_{4}\right)$, and concentrated under reduced pressure. The residue was presorbed on silica gel and purified by column chromatography $(10 \rightarrow 20 \%$ EtOAc/hexanes) to afford exclusively anti-addition products 7 as viscous oils in quantitative yields.<smiles>C=CC(Cc1ccccc1)[C@H](OCC(C)=O)C(OCc1ccccc1)c1ccccc1</smiles>

Compound 7a. 97\%; $R_{f} 0.66$ (25\% EtOAc/hexanes); ${ }^{1} \mathrm{H}$ NMR $\left(\mathrm{CDCl}_{3}\right): \delta 7.46-7.23(\mathrm{~m}, 20 \mathrm{H}), 6.01$ $(\mathrm{m}, 1 \mathrm{H}), 5.44(\mathrm{br} \mathrm{d}, J=9.4 \mathrm{~Hz}, 1 \mathrm{H}), 5.39(\mathrm{br} \mathrm{d}, J=17.1 \mathrm{~Hz}, 1 \mathrm{H}), 4.90(\mathrm{~d}, J=11.6 \mathrm{~Hz}, 1 \mathrm{H}), 4.89(\mathrm{~d}$, $J=11.3 \mathrm{~Hz}, 1 \mathrm{H}), 4.74(\mathrm{~d}, J=11.8 \mathrm{~Hz}, 1 \mathrm{H}), 4.67(\mathrm{~d}, J=11.6 \mathrm{~Hz}, 1 \mathrm{H}), 4.57(\mathrm{~d}, J=11.3 \mathrm{~Hz}, 1 \mathrm{H})$, $4.44(\mathrm{~d}, J=11.8 \mathrm{~Hz}, 1 \mathrm{H}), 4.11(\psi \mathrm{t}, J=5.8 \mathrm{~Hz}, 1 \mathrm{H}), 4.04(\mathrm{dd}, J=6.9,5.2 \mathrm{~Hz}, 1 \mathrm{H}), 3.80(\mathrm{~m}, 1 \mathrm{H})$, $3.55(\mathrm{~m}, 1 \mathrm{H}), 3.54(\mathrm{~s}, 3 \mathrm{H}), 3.09(\mathrm{dd}, J=15.7,4.4 \mathrm{~Hz}, 1 \mathrm{H}), 2.86(\mathrm{dd}, J=15.7,10.2 \mathrm{~Hz}, 1 \mathrm{H}) ;{ }^{13} \mathrm{C}$ NMR $\left(\mathrm{CDCl}_{3}\right): \delta 173.1,141.9,139.1,139.0,138.4,135.6,128.7,128.6,128.5,128.3,128.0,127.9$, 127.8, 127.6, 127.6, 127.0, 119.2, 82.4, 82.3, 81.5, 75.0, 73.4, 70.9, 51.6, 43.6, 36.7; HRMS m/z (EI) calcd for $\mathrm{C}_{36} \mathrm{H}_{39} \mathrm{O}_{5}(\mathrm{M}+\mathrm{H})^{+}$551.2798, found 551.2795. 


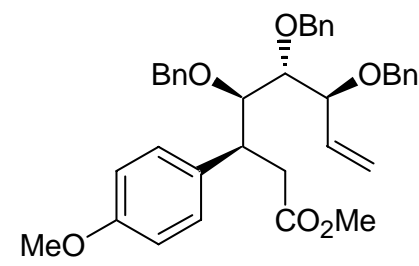

Compound 7b. 95\%; $R_{f} 0.54$ (25\% EtOAc/hexanes); ${ }^{1} \mathrm{H}$ NMR $\left(\mathrm{CDCl}_{3}\right): \delta 7.41-7.36(\mathrm{~m}, 15 \mathrm{H}), 7.09$ $(\mathrm{d}, J=8.5 \mathrm{~Hz}, 2 \mathrm{H}), 6.83(\mathrm{~d}, J=8.5 \mathrm{~Hz}, 2 \mathrm{H}), 5.95(\mathrm{~m}, 1 \mathrm{H}), 5.39(\mathrm{br} \mathrm{d}, J=9.5 \mathrm{~Hz}, 1 \mathrm{H}), 5.34(\mathrm{br} \mathrm{d}, J$ $=17.9 \mathrm{~Hz}, 1 \mathrm{H}), 4.85(\mathrm{~d}, J=11.6 \mathrm{~Hz}, 1 \mathrm{H}), 4.83(\mathrm{~d}, J=11.3 \mathrm{~Hz}, 1 \mathrm{H}), 4.68(\mathrm{~d}, J=11.8 \mathrm{~Hz}, 1 \mathrm{H}), 4.61$ $(\mathrm{d}, J=11.6 \mathrm{~Hz}, 1 \mathrm{H}), 4.54(\mathrm{~d}, J=11.3 \mathrm{~Hz}, 1 \mathrm{H}), 4.39(\mathrm{~d}, J=11.8 \mathrm{~Hz}, 1 \mathrm{H}), 4.06(\psi \mathrm{t}, J=6.1 \mathrm{~Hz}, 1 \mathrm{H})$, $3.93(\mathrm{dd}, J=7.4,5.2 \mathrm{~Hz}, 1 \mathrm{H}), 3.82(\mathrm{~s}, 3 \mathrm{H}), 3.68(\mathrm{~m}, 1 \mathrm{H}), 3.51(\mathrm{~s}, 3 \mathrm{H}), 3.49(\mathrm{~m}, 1 \mathrm{H}), 3.00(\mathrm{dd}, J=$ 15.4, $4.4 \mathrm{~Hz}, 1 \mathrm{H}), 2.75(\mathrm{dd}, J=15.4,10.5 \mathrm{~Hz}, 1 \mathrm{H}) ;{ }^{13} \mathrm{C} \mathrm{NMR}\left(\mathrm{CDCl}_{3}\right): \delta 173.2,158.5,139.1,139.0$, 138.4, 135.6, 133.7, 129.4, 128.5, 128.4, 128.2, 127.9, 127.8, 127.7, 127.5, 119.1, 114.0, 82.4, 81.6, 75.0, 73.2, 70.8, 55.3, 51.5, 42.8, 36.9; HRMS $m / z$ (EI) calcd for $\mathrm{C}_{37} \mathrm{H}_{41} \mathrm{O}_{6}(\mathrm{M}+\mathrm{H})^{+} 581.2903$, found 581.2910 .

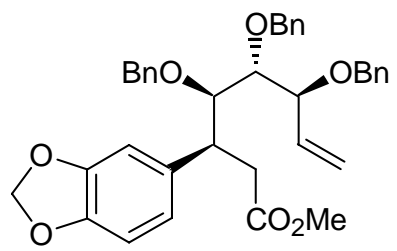

Compound 7c. $95 \% ;{ }^{1} \mathrm{H}$ NMR $\left(\mathrm{CDCl}_{3}\right): \delta 7.38-7.33(\mathrm{~m}, 15 \mathrm{H}), 6.70(\mathrm{~d}, J=8.0 \mathrm{~Hz}, 1 \mathrm{H}), 6.64$ (br s, $1 \mathrm{H}), 6.59(\mathrm{br} \mathrm{d}, J=8.0 \mathrm{~Hz}, 1 \mathrm{H}), 5.93(\mathrm{~s}, 2 \mathrm{H}), 5.92(\mathrm{~m}, 1 \mathrm{H}), 5.38(\mathrm{br} \mathrm{d}, J=5.2 \mathrm{~Hz}, 1 \mathrm{H}), 5.34$ (br d, $J$ $=13.7 \mathrm{~Hz}, 1 \mathrm{H}), 4.83(\mathrm{~d}, J=11.8 \mathrm{~Hz}, 1 \mathrm{H}), 4.78(\mathrm{~d}, J=11.3 \mathrm{~Hz}, 1 \mathrm{H}), 4.65(\mathrm{~d}, J=11.6 \mathrm{~Hz}, 1 \mathrm{H}), 4.59$ $(\mathrm{d}, J=11.6 \mathrm{~Hz}, 1 \mathrm{H}), 4.50(\mathrm{~d}, J=11.3 \mathrm{~Hz}, 1 \mathrm{H}), 4.37(\mathrm{~d}, J=11.8 \mathrm{~Hz}, 1 \mathrm{H}), 4.07(\psi \mathrm{t}, J=6.1 \mathrm{~Hz}, 1 \mathrm{H})$, $3.86(\mathrm{dd}, J=6.9,5.2 \mathrm{~Hz}, 1 \mathrm{H}), 3.60(\mathrm{~m}, 1 \mathrm{H}), 3.51(\mathrm{~s}, 3 \mathrm{H}), 3.47(\psi \mathrm{t}, J=5.2 \mathrm{~Hz}, 1 \mathrm{H}), 2.93(\mathrm{dd}, J=$ 15.7, $4.4 \mathrm{~Hz}, 1 \mathrm{H}), 2.69(\mathrm{dd}, J=15.7,10.5 \mathrm{~Hz}, 1 \mathrm{H}) ;{ }^{13} \mathrm{C} \mathrm{NMR}\left(\mathrm{CDCl}_{3}\right): \delta 173.0,147.7,146.4,138.9$, $138.8,138.2,135.5,135.4,128.5,128.4,128.2$, 127.9, 127.8, 127.7, 127.5, 121.6, 119.2, 108.6, 108.3, 101.0, 82.3, 82.2, 81.5, 74.9, 73.2, 70.8, 51.5, 43.0, 36.7; HRMS $m / z$ (EI) calcd for $\mathrm{C}_{37} \mathrm{H}_{38} \mathrm{O}_{7}$ $(\mathrm{M})^{+}$594.2618, found 594.2612.<smiles>C=CC(Cc1ccccc1)[C@H](OCCCC)[C@H](OC(C)=O)c1cc(OC)c2c(c1)OCO2</smiles>

Compound 7d. 97\%; $R_{f} 0.42\left(25 \%\right.$ EtOAc/hexanes); ${ }^{1} \mathrm{H}$ NMR $\left(\mathrm{CDCl}_{3}\right): \delta 7.36-7.28(\mathrm{~m}, 15 \mathrm{H})$, $6.33(\mathrm{~s}, 1 \mathrm{H}), 6.32(\mathrm{~s}, 1 \mathrm{H}), 5.94(\mathrm{br} \mathrm{s}, 2 \mathrm{H}), 5.93(\mathrm{~m}, 1 \mathrm{H}), 5.38(\mathrm{~s}, 1 \mathrm{H}), 5.34(\mathrm{br} \mathrm{d}, J=6.6 \mathrm{~Hz}, 1 \mathrm{H})$, $4.84(\mathrm{~d}, J=11.8 \mathrm{~Hz}, 1 \mathrm{H}), 4.78(\mathrm{~d}, J=11.3 \mathrm{~Hz}, 1 \mathrm{H}), 4.65(\mathrm{~d}, J=11.6 \mathrm{~Hz}, 1 \mathrm{H}), 4.56(\mathrm{~d}, J=11.6 \mathrm{~Hz}$, $1 \mathrm{H}), 4.46(\mathrm{~d}, J=11.3 \mathrm{~Hz}, 1 \mathrm{H}), 4.37(\mathrm{~d}, J=11.8 \mathrm{~Hz}, 1 \mathrm{H}), 4.08(\psi \mathrm{t}, J=5.8 \mathrm{~Hz}, 1 \mathrm{H}), 3.86(\mathrm{dd}, J=$ 6.3, $5.5 \mathrm{~Hz}, 1 \mathrm{H}), 3.65(\mathrm{~s}, 3 \mathrm{H}), 3.59(\mathrm{~m}, 1 \mathrm{H}), 3.51(\mathrm{~s}, 3 \mathrm{H}), 3.48(\psi \mathrm{t}, J=5.5 \mathrm{~Hz}, 1 \mathrm{H}), 2.90(\mathrm{dd}, J=$ $15.7,4.7 \mathrm{~Hz}, 1 \mathrm{H}), 2.70(\mathrm{dd}, J=15.7,10.5 \mathrm{~Hz}, 1 \mathrm{H}) ;{ }^{13} \mathrm{C} \mathrm{NMR}\left(\mathrm{CDCl}_{3}\right): \delta 173.0,148.9,143.5,139.0$, $138.9,138.2$, 136.4, 135.4, 134.0, 128.5, 128.4, 128.1, 127.7, 127.6, 127.5, 119.1, 107.8, 102.1, 
101.4, 82.5, 82.4, 81.5, 74.9, 73.2, 70.9, 56.4, 51.6, 43.2, 36.4; HRMS $m / z$ (EI) calcd for $\mathrm{C}_{38} \mathrm{H}_{40} \mathrm{O}_{8}$ $(\mathrm{M})^{+} 624.2723$, found 624.2715 .

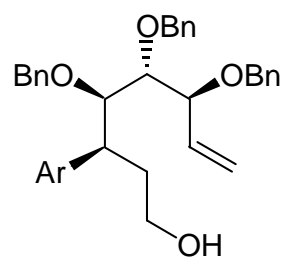

\section{General procedure for ester reduction.}

To the solution of an appropriate ester 7a-d $(3 \mathrm{mmol})$ in ether $(50 \mathrm{~mL})$ cooled to $0{ }^{\circ} \mathrm{C}$ was added $\mathrm{LiAlH}_{4}(0.23 \mathrm{~g}, 6 \mathrm{mmol})$ in one portion. The reaction mixture was stirred for $3 \mathrm{~h}$ while it was allowed to warm up to rt. Careful quenching with sat. $\mathrm{NH}_{4} \mathrm{Cl}(10 \mathrm{~mL})$ was followed by extraction with ether $(3 \times 100 \mathrm{~mL})$. Organic layers were combined, dried $\left(\mathrm{MgSO}_{4}\right)$ and concentrated under reduced pressure to afford the corresponding primary alcohols as viscous colorless oils. The reduction products $\mathbf{7}$ 'a-d were used without purification in the next step. Small portions of each crude alcohol were purified (35\% EtOAc/hexanes) for characterization.<smiles>C=C[C@@H](CCO)[C@@H](OCCCC)[C@@H](OCc1ccccc1)C(CCO)c1ccccc1</smiles>

Compound 7'a. $R_{f} 0.30$ (25\% EtOAc/hexanes); ${ }^{1} \mathrm{H}$ NMR $\left(\mathrm{CDCl}_{3}\right): \delta 7.35-7.12(\mathrm{~m}, 20 \mathrm{H}), 5.91(\mathrm{~m}$, $1 \mathrm{H}), 5.36(\mathrm{br} \mathrm{d}, J=8.8 \mathrm{~Hz}, 1 \mathrm{H}), 5.31(\mathrm{br} \mathrm{d}, J=16.5 \mathrm{~Hz}, 1 \mathrm{H}), 4.78(\mathrm{~d}, J=11.4 \mathrm{~Hz}, 1 \mathrm{H}), 4.77(\mathrm{~d}, J=$ $11.2 \mathrm{~Hz}, 1 \mathrm{H}), 4.64(\mathrm{~d}, J=11.8 \mathrm{~Hz}, 1 \mathrm{H}), 4.54(\mathrm{~d}, J=11.4 \mathrm{~Hz}, 1 \mathrm{H}), 4.47(\mathrm{~d}, J=11.2 \mathrm{~Hz}, 1 \mathrm{H}), 4.36$ $(\mathrm{d}, J=11.8 \mathrm{~Hz}, 1 \mathrm{H}), 4.05(\psi \mathrm{t}, J=5.5 \mathrm{~Hz}, 1 \mathrm{H}), 3.88(\mathrm{dd}, J=6.6,5.4 \mathrm{~Hz}, 1 \mathrm{H}), 3.49(\psi \mathrm{t}, J=6.2 \mathrm{~Hz}$, $1 \mathrm{H}), 3.46(\mathrm{~m}, 1 \mathrm{H}), 3.22(\mathrm{~m}, 1 \mathrm{H}), 3.20(\mathrm{~m}, 1 \mathrm{H}), 2.18(\mathrm{~m}, 1 \mathrm{H}), 2.00(\mathrm{~m}, 1 \mathrm{H}), 1.35(\mathrm{br} \mathrm{s}, 1 \mathrm{H}) ;{ }^{13} \mathrm{C}$ NMR $\left(\mathrm{CDCl}_{3}\right): \delta 142.4,139.1,138.2,135.5,128.7,128.5,128.4,128.4,127.9,127.8,127.5,127.4$, $126.7,119.0,83.2,82.7,81.3,74.9,73.4,70.8,61.2,43.4,33.7$; HRMS $m / z$ (EI) calcd for $\mathrm{C}_{35} \mathrm{H}_{39} \mathrm{O}_{4}$ $(\mathrm{M}+\mathrm{H})^{+}$523.2848, found 523.2848.<smiles>C=CC(CCO)[C@H](OCCCC)[C@@H](OCc1ccccc1)C(CCO)c1ccc(OC)cc1</smiles>

Compound 7'b. $R_{f} 0.33$ (25\% EtOAc/hexanes); ${ }^{1} \mathrm{H}$ NMR $\left(\mathrm{CDCl}_{3}\right): \delta 7.38-7.29(\mathrm{~m}, 15 \mathrm{H}), 7.04$ (d, $J$ $=8.5 \mathrm{~Hz}, 2 \mathrm{H}), 6.81(\mathrm{~d}, J=8.5 \mathrm{~Hz}, 2 \mathrm{H}), 5.91(\mathrm{~m}, 1 \mathrm{H}), 5.36(\mathrm{br} \mathrm{d}, J=8.8 \mathrm{~Hz}, 1 \mathrm{H}), 5.32(\mathrm{br} \mathrm{d}, J=$ $16.0 \mathrm{~Hz}, 1 \mathrm{H}), 4.80(\mathrm{~d}, J=11.6 \mathrm{~Hz}, 1 \mathrm{H}), 4.77(\mathrm{~d}, J=11.0 \mathrm{~Hz}, 1 \mathrm{H}), 4.65(\mathrm{~d}, J=11.8 \mathrm{~Hz}, 1 \mathrm{H}), 4.55$ $(\mathrm{d}, J=11.6 \mathrm{~Hz}, 1 \mathrm{H}), 4.50(\mathrm{~d}, J=11.0 \mathrm{~Hz}, 1 \mathrm{H}), 4.37(\mathrm{~d}, J=11.8 \mathrm{~Hz}, 1 \mathrm{H}), 4.06(\psi \mathrm{t}, J=5.8 \mathrm{~Hz}, 1 \mathrm{H})$, $3.84(\mathrm{dd}, J=6.6,5.2 \mathrm{~Hz}, 1 \mathrm{H}), 3.80(\mathrm{~s}, 3 \mathrm{H}), 3.49(\psi \mathrm{t}, J=5.2 \mathrm{~Hz}, 1 \mathrm{H}), 3.46(\mathrm{~m}, 1 \mathrm{H}), 3.32(\mathrm{~m}, 1 \mathrm{H})$, $3.16(\mathrm{~m}, 1 \mathrm{H}), 2.16(\mathrm{~m}, 1 \mathrm{H}), 1.95(\mathrm{~m}, 1 \mathrm{H}), 1.58(\mathrm{br} \mathrm{s}, 1 \mathrm{H}) ;{ }^{13} \mathrm{C} \mathrm{NMR}\left(\mathrm{CDCl}_{3}\right): \delta 158.4,139.1,138.2$, 
$135.5,134.3,129.6,128.5,128.4,128.4,127.9,127.8,127.7,127.5,127.4,119.0,114.0,83.3,82.7$, 81.3, 74.9, 73.4, 70.8, 61.2, 55.3, 42.6, 33.9; HRMS $m / z$ (EI) calcd for $\mathrm{C}_{36} \mathrm{H}_{41} \mathrm{O}_{5}(\mathrm{M}+\mathrm{H})^{+}$553.2954, found 553.2947.<smiles>C=CC(CCO)[C@@H](OCCCC)[C@@H](OCc1ccccc1)[C@@H](CCO)c1ccc2c(c1)OCO2</smiles>

Compound 7'c. $R_{f} 0.12\left(25 \%\right.$ EtOAc/hexanes); ${ }^{1} \mathrm{H}$ NMR $\left(\mathrm{CDCl}_{3}\right): \delta 7.38-7.29(\mathrm{~m}, 15 \mathrm{H}), 6.71(\mathrm{~d}, J$ $=7.9 \mathrm{~Hz}, 1 \mathrm{H}), 6.65(\mathrm{~d}, J=1.5 \mathrm{~Hz}, 1 \mathrm{H}), 6.56(\mathrm{dd}, J=8.0,1.5 \mathrm{~Hz}, 1 \mathrm{H}), 5.93(\mathrm{~s}, 2 \mathrm{H}), 5.92(\mathrm{~m}, 1 \mathrm{H})$, 5.39 (br d, $J=3.2 \mathrm{~Hz}, 1 \mathrm{H}), 5.34(\mathrm{br} \mathrm{d}, J=10.5 \mathrm{~Hz}, 1 \mathrm{H}), 4.81(\mathrm{~d}, J=11.6 \mathrm{~Hz}, 1 \mathrm{H}), 4.77(\mathrm{~d}, J=11.0$ $\mathrm{Hz}, 1 \mathrm{H}), 4.65(\mathrm{~d}, J=11.8 \mathrm{~Hz}, 1 \mathrm{H}), 4.56(\mathrm{~d}, J=11.6 \mathrm{~Hz}, 1 \mathrm{H}), 4.51(\mathrm{~d}, J=11.0 \mathrm{~Hz}, 1 \mathrm{H}), 4.38(\mathrm{~d}, J=$ $11.8 \mathrm{~Hz}, 1 \mathrm{H}), 4.09(\psi \mathrm{t}, J=5.8 \mathrm{~Hz}, 1 \mathrm{H}), 3.81(\psi \mathrm{t}, J=5.4 \mathrm{~Hz}, 1 \mathrm{H}), 3.52(\psi \mathrm{t}, J=5.4 \mathrm{~Hz}, 1 \mathrm{H}), 3.48$ $(\mathrm{m}, 1 \mathrm{H}), 3.32(\mathrm{~m}, 1 \mathrm{H}), 3.12(\mathrm{~m}, 1 \mathrm{H}), 2.13(\mathrm{~m}, 1 \mathrm{H}), 1.90(\mathrm{~m}, 1 \mathrm{H}), 1.40(\mathrm{~m}, 1 \mathrm{H}) ;{ }^{13} \mathrm{C} \mathrm{NMR}\left(\mathrm{CDCl}_{3}\right)$ : $\delta 147.9,146.3,139.1,139.0,138.2,136.2,135.4,128.5,128.4,127.8,127.5,127.4,121.8,119.0$, $108.7,108.3,101.0,83.3,82.6,81.3,74.9,73.5,70.8,61.1,43.0,33.8$; HRMS $m / z$ (EI) calcd for $\mathrm{C}_{36} \mathrm{H}_{38} \mathrm{O}_{6}(\mathrm{M})^{+}$566.2668, found 566.2663.

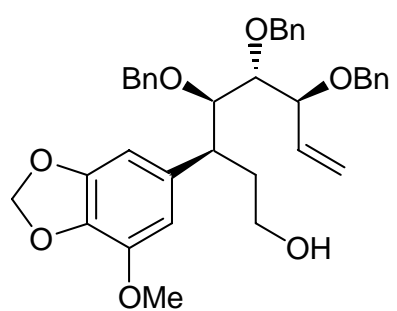

Compound 7'd. $R_{f} 0.30$ (25\% EtOAc/hexanes); ${ }^{1} \mathrm{H}$ NMR $\left(\mathrm{CDCl}_{3}\right): \delta 7.35-7.28(\mathrm{~m}, 15 \mathrm{H}), 6.33$ (s, $1 \mathrm{H}), 6.32(\mathrm{~s}, 1 \mathrm{H}), 5.94(\mathrm{~s}, 2 \mathrm{H}), 5.93(\mathrm{~m}, 1 \mathrm{H}), 5.39(\mathrm{~m}, 1 \mathrm{H}), 5.35(\mathrm{br} \mathrm{d}, J=5.2 \mathrm{~Hz}, 1 \mathrm{H}), 4.83(\mathrm{~d}, J=$ $11.6 \mathrm{~Hz}, 1 \mathrm{H}), 4.79(\mathrm{~d}, J=11.3 \mathrm{~Hz}, 1 \mathrm{H}), 4.65(\mathrm{~d}, J=11.8 \mathrm{~Hz}, 1 \mathrm{H}), 4.55(\mathrm{~d}, J=11.6 \mathrm{~Hz}, 1 \mathrm{H}), 4.48$ $(\mathrm{d}, J=11.3 \mathrm{~Hz}, 1 \mathrm{H}), 4.38(\mathrm{~d}, J=11.8 \mathrm{~Hz}, 1 \mathrm{H}), 4.11(\psi \mathrm{t}, J=5.5 \mathrm{~Hz}, 1 \mathrm{H}), 3.82(\psi \mathrm{t}, J=5.8 \mathrm{~Hz}, 1 \mathrm{H})$, $3.65(\mathrm{~s}, 3 \mathrm{H}), 3.54(\psi \mathrm{t}, J=5.5 \mathrm{~Hz}, 1 \mathrm{H}), 3.49(\mathrm{~m}, 1 \mathrm{H}), 3.34(\mathrm{~m}, 1 \mathrm{H}), 3.12(\mathrm{~m}, 1 \mathrm{H}), 2.11(\mathrm{~m}, 1 \mathrm{H}), 1.92$ $(\mathrm{m}, 1 \mathrm{H}), 1.38(\mathrm{br} \mathrm{s}, 1 \mathrm{H}) ;{ }^{13} \mathrm{C}$ NMR $\left(\mathrm{CDCl}_{3}\right): \delta 149.0,143.6,139.1,138.2,137.1,135.4,133.9$, 128.5, 128.4, 128.3, 127.8, 127.6, 127.5, 127.4, 119.0, 107.9, 102.3, 101.4, 83.4, 82.8, 81.3, 74.9, 73.4, 70.8, 61.1, 56.4, 43.2, 33.7; HRMS m/z (EI) calcd for $\mathrm{C}_{37} \mathrm{H}_{40} \mathrm{O}_{7}(\mathrm{M})^{+}$596.2774, found 596.2775 .<smiles>C=CC([Al])C(OCc1ccccc1)[C@@H](OCc1ccccc1)C(=O)c1ccccc1</smiles>

General procedure for the terminal double bond installation by selenoxide elimination.

To the solution of an appropriate crude alcohol 7'a-d $(3 \mathrm{mmol})$ and $o$-nitrophenylselenocyanate $(0.75 \mathrm{~g}, 3.3 \mathrm{mmol})$ in THF $(30 \mathrm{~mL})$ was added tributylphosphine $(0.67 \mathrm{~g}, 3.3 \mathrm{mmol})$ at $\mathrm{rt}$. The 
reaction mixture immediately turned deep brown and TLC showed that it was complete. The mixture was quenched with $1 \mathrm{M} \mathrm{NaOH}(20 \mathrm{~mL})$ and stirred for $30 \mathrm{~min}$. The mixture was diluted with water $(100 \mathrm{~mL})$ and extracted with ether $(3 \times 100 \mathrm{~mL})$. Organic layers were combined, dried $\left(\mathrm{MgSO}_{4}\right)$ and concentrated under reduced pressure. The yellow residue was dissolved in THF (30 $\mathrm{mL})$, cooled down to $0{ }^{\circ} \mathrm{C}$, and $30 \% \mathrm{H}_{2} \mathrm{O}_{2}(3.4 \mathrm{~g}, 30 \mathrm{mmol})$ was added to the solution. After an overnight stirring at $\mathrm{rt}$ the reaction mixture was quenched with $\mathrm{NaHSO}_{3}$ and extracted with ether $(3 \times 100 \mathrm{~mL})$. Organic layers were combined, dried $\left(\mathrm{MgSO}_{4}\right)$ and concentrated under reduced pressure. The residue was presorbed on silica gel and purified by column chromatography $(5 \% \rightarrow 10 \%$, EtOAc/hexanes) to afford pure dienes $\mathbf{8}$ as colorless oils.<smiles>C=CC(c1ccccc1)[C@H](OCc1ccccc1)[C@@H](OCc1ccccc1)c1ccccc1</smiles>

Compound 8a. 75\% (3 steps); $R_{f} 0.73$ (25\% EtOAc/hexanes); ${ }^{1} \mathrm{H}$ NMR $\left(\mathrm{CDCl}_{3}\right): \delta 7.44-7.21$ (m, $20 \mathrm{H}), 6.40(\mathrm{~m}, 1 \mathrm{H}), 6.01(\mathrm{~m}, 1 \mathrm{H}), 5.39$ (br d, $J=8.5 \mathrm{~Hz}, 1 \mathrm{H}), 5.36$ (br d, $J=17.3 \mathrm{~Hz}, 1 \mathrm{H}), 5.20$ (br $\mathrm{d}, J=10.5 \mathrm{~Hz}, 1 \mathrm{H}), 5.00(\mathrm{br} \mathrm{d}, J=17.1 \mathrm{~Hz}, 1 \mathrm{H}), 4.77(\mathrm{~d}, J=11.3 \mathrm{~Hz}, 1 \mathrm{H}), 4.74(\mathrm{~d}, J=11.8 \mathrm{~Hz}$, $1 \mathrm{H}), 4.68(\mathrm{~d}, J=10.7 \mathrm{~Hz}, 1 \mathrm{H}), 4.67(\mathrm{~d}, J=11.3 \mathrm{~Hz}, 1 \mathrm{H}), 4.44(\mathrm{~d}, J=11.8 \mathrm{~Hz}, 1 \mathrm{H}), 4.27(\mathrm{~d}, J=10.7$ $\mathrm{Hz}, 1 \mathrm{H}), 4.11(\mathrm{~m}, 2 \mathrm{H}), 3.59(\mathrm{dd}, J=9.4,5.2 \mathrm{~Hz}, 1 \mathrm{H}), 3.52(\psi \mathrm{t}, J=5.0 \mathrm{~Hz}, 1 \mathrm{H}) ;{ }^{13} \mathrm{C} \mathrm{NMR}\left(\mathrm{CDCl}_{3}\right)$ : $\delta 142.6,139.1,138.1,137.8,136.1,128.8,128.5,128.4,128.3,128.2,128.0,127.9,127.5,127.4$, $126.5,119.0,117.4,83.5,83.1,80.0,75.4,74.2,70.4,51.2 ; \mathrm{HRMS} \mathrm{m} / \mathrm{z}$ (EI) calcd for $\mathrm{C}_{35} \mathrm{H}_{37} \mathrm{O}_{3}$ $(\mathrm{M}+\mathrm{H})^{+}$505.2743, found 505.2757.

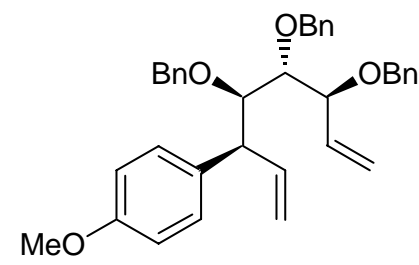

Compound 8b. 84\% (3 steps); $R_{f} 0.69$ (25\% EtOAc/hexanes); ${ }^{1} \mathrm{H}$ NMR $\left(\mathrm{CDCl}_{3}\right): \delta 7.36-7.18(\mathrm{~m}$, $15 \mathrm{H}), 7.07(\mathrm{~d}, J=8.5 \mathrm{~Hz}, 2 \mathrm{H}), 6.82(\mathrm{~d}, J=8.5 \mathrm{~Hz}, 2 \mathrm{H}), 6.31(\mathrm{~m}, 1 \mathrm{H}), 5.95(\mathrm{~m}, 1 \mathrm{H}), 5.34(\mathrm{br} \mathrm{d}, J=$ $8.5 \mathrm{~Hz}, 1 \mathrm{H}), 5.30(\mathrm{br} \mathrm{d}, J=15.4 \mathrm{~Hz}, 1 \mathrm{H}), 5.13(\mathrm{dd}, J=10.5,1.7 \mathrm{~Hz}, 1 \mathrm{H}), 4.93(\mathrm{dd}, J=17.1,1.7 \mathrm{~Hz}$, $1 \mathrm{H}), 4.72(\mathrm{~d}, J=11.3 \mathrm{~Hz}, 1 \mathrm{H}), 4.68(\mathrm{~d}, J=11.8 \mathrm{~Hz}, 1 \mathrm{H}), 4.63(\mathrm{~d}, J=11.0 \mathrm{~Hz}, 1 \mathrm{H}), 4.60(\mathrm{~d}, J=11.3$ $\mathrm{Hz}, 1 \mathrm{H}), 4.38(\mathrm{~d}, J=11.8 \mathrm{~Hz}, 1 \mathrm{H}), 4.27(\mathrm{~d}, J=11.0 \mathrm{~Hz}, 1 \mathrm{H}), 4.06(\mathrm{dd}, J=7.2,4.7 \mathrm{~Hz}, 1 \mathrm{H}), 3.98$ $(\psi \mathrm{t}, J=5.8 \mathrm{~Hz}, 1 \mathrm{H}), 3.80(\mathrm{~s}, 3 \mathrm{H}), 3.50(\mathrm{dd}, J=9.4,5.5 \mathrm{~Hz}, 1 \mathrm{H}), 3.45(\psi \mathrm{t}, J=6.1 \mathrm{~Hz}, 1 \mathrm{H}) ;{ }^{13} \mathrm{C}$ NMR $\left(\mathrm{CDCl}_{3}\right): \delta 158.2,139.1,138.1,136.1,134.7,129.3,128.7,128.5,128.3,128.2,128.0,127.9$, 127.4, 127.3, 118.9, 117.0, 113.9, 83.4, 83.0, 80.2, 75.4, 74.1, 70.4, 55.3, 50.3; HRMS m/z (EI) calcd for $\mathrm{C}_{36} \mathrm{H}_{38} \mathrm{O}_{4}(\mathrm{M})^{+}$534.2770, found 534.2757. 


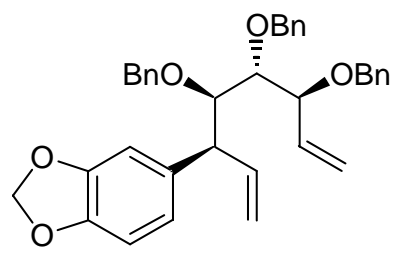

Compound 8c. 77\% (3 steps); $R_{f} 0.69$ (25\% EtOAc/hexanes); ${ }^{1} \mathrm{H}$ NMR $\left(\mathrm{CDCl}_{3}\right): \delta 7.35-7.17$ (m, $15 \mathrm{H}), 6.70(\mathrm{~d}, J=7.9 \mathrm{~Hz}, 1 \mathrm{H}), 6.64(\mathrm{~d}, J=1.5 \mathrm{~Hz}, 1 \mathrm{H}), 6.57(\mathrm{dd}, J=7.9,1.5 \mathrm{~Hz}, 1 \mathrm{H}), 6.25(\mathrm{~m}$, $1 \mathrm{H}), 5.93(\mathrm{~m}, 1 \mathrm{H}), 5.92(\mathrm{~s}, 2 \mathrm{H}), 5.33(\mathrm{dd}, J=8.4,1.9 \mathrm{~Hz}, 1 \mathrm{H}), 5.29(\mathrm{br} \mathrm{d}, J=13.5 \mathrm{~Hz}, 1 \mathrm{H}), 5.11$ $(\mathrm{dd}, J=10.3,1.9 \mathrm{~Hz}, 1 \mathrm{H}), 4.92(\mathrm{dd}, J=17.2,1.9 \mathrm{~Hz}, 1 \mathrm{H}), 4.71(\mathrm{~d}, J=11.4 \mathrm{~Hz}, 1 \mathrm{H}), 4.66(\mathrm{~d}, J=$ $11.8 \mathrm{~Hz}, 1 \mathrm{H}), 4.62(\mathrm{~d}, J=10.9 \mathrm{~Hz}, 1 \mathrm{H}), 4.60(\mathrm{~d}, J=11.4 \mathrm{~Hz}, 1 \mathrm{H}), 4.36(\mathrm{~d}, J=11.8 \mathrm{~Hz}, 1 \mathrm{H}), 4.29$ $(\mathrm{d}, J=10.9 \mathrm{~Hz}, 1 \mathrm{H}), 4.04(\mathrm{dd}, J=7.5,4.7 \mathrm{~Hz}, 1 \mathrm{H}), 3.94(\psi \mathrm{t}, J=5.8 \mathrm{~Hz}, 1 \mathrm{H}), 3.44(\mathrm{~m}, 2 \mathrm{H}) ;{ }^{13} \mathrm{C}$ NMR $\left(\mathrm{CDCl}_{3}\right): \delta 147.6,146.0,139.0,138.1,137.9,136.5,136.0,128.7,128.5,128.3,128.2,128.0$, 127.9, 127.5, 127.4, 121.3, 118.9, 117.2, 108.8, 108.3, 100.9, 83.3, 83.0, 80.1, 75.4, 74.2, 70.4, 50.7; HRMS $m / z$ (EI) calcd for $\mathrm{C}_{36} \mathrm{H}_{36} \mathrm{O}_{5}(\mathrm{M})^{+} 548.2563$, found 548.2562 .<smiles>C=CC(OCc1ccccc1)[C@H](OCc1ccccc1)[C@@H](OCc1ccccc1)c1ccccc1</smiles>

Compound 8d. 84\% (3 steps); $R_{f} 0.65$ (25\% EtOAc/hexanes); ${ }^{1} \mathrm{H}$ NMR $\left(\mathrm{CDCl}_{3}\right): \delta 7.39-7.19(\mathrm{~m}$, $15 \mathrm{H}), 6.36(\mathrm{~d}, J=1.4 \mathrm{~Hz}, 1 \mathrm{H}), 6.34(\mathrm{~d}, J=1.4 \mathrm{~Hz}, 1 \mathrm{H}), 6.28(\mathrm{~m}, 1 \mathrm{H}), 5.98(\mathrm{~m}, 1 \mathrm{H}), 5.94(\mathrm{~s}, 2 \mathrm{H})$, 5.37 (br d, $J=3.3 \mathrm{~Hz}, 1 \mathrm{H}), 5.32$ (br d, $J=10.5 \mathrm{~Hz}, 1 \mathrm{H}), 5.16$ (dd, $J=10.2,1.9 \mathrm{~Hz}, 1 \mathrm{H}), 4.97$ (dd, $J$ $=17.3,1.9 \mathrm{~Hz}, 1 \mathrm{H}), 4.75(\mathrm{~d}, J=11.6 \mathrm{~Hz}, 1 \mathrm{H}), 4.69(\mathrm{~d}, J=11.8 \mathrm{~Hz}, 1 \mathrm{H}), 4.67(\mathrm{~d}, J=10.7 \mathrm{~Hz}, 1 \mathrm{H})$, $4.63(\mathrm{~d}, J=11.6 \mathrm{~Hz}, 1 \mathrm{H}), 4.39(\mathrm{~d}, J=11.8 \mathrm{~Hz}, 1 \mathrm{H}), 4.30(\mathrm{~d}, J=10.7 \mathrm{~Hz}, 1 \mathrm{H}), 4.08(\mathrm{dd}, J=7.4,4.4$ $\mathrm{Hz}, 1 \mathrm{H}), 3.97$ (dd, $J=6.6,5.0 \mathrm{~Hz}, 1 \mathrm{H}), 3.69$ (s, 3H), 3.49 (dd, $J=6.6,4.4 \mathrm{~Hz}, 1 \mathrm{H}), 3.45$ (dd, 1H); ${ }^{13} \mathrm{C}$ NMR $\left(\mathrm{CDCl}_{3}\right): \delta 148.8,143.5,139.0,138.1,137.6,137.3,136.0,133.7,128.6,128.5,128.3$, $128.2,127.9,127.8,127.4,127.3,118.9,117.4,107.6,102.4,101.3,83.5,83.1,80.1,75.4,74.2$, 70.4, 56.4, 51.0; HRMS $m / z$ (EI) calcd for $\mathrm{C}_{37} \mathrm{H}_{38} \mathrm{O}_{6}(\mathrm{M})^{+}$578.2668, found 578.2677.

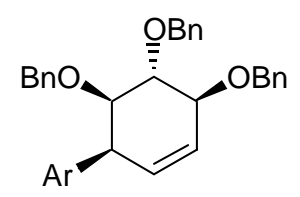

\section{General procedure for ring-closing metathesis.}

To the solution of an appropriate diene 8a-d $(2 \mathrm{mmol})$ in dry $\mathrm{CH}_{2} \mathrm{Cl}_{2}(100 \mathrm{~mL})$ was added $\left(\mathrm{Cy}_{3} \mathrm{P}\right)_{2}(\mathrm{PhCH}=) \mathrm{RuCl}_{2}(0.08 \mathrm{~g}, 0.1 \mathrm{mmol})$. After an overnight stirring DMSO $(0.78 \mathrm{~g}, 10 \mathrm{mmol})$ was added and the reaction mixture was stirred for additional $6 \mathrm{~h}$. The mixture was concentrated under reduced pressure and the residue was presorbed on silica gel and purified by column chromatography $(10 \% \rightarrow 15 \%$ EtOAc/hexanes) to afford pure conduritols 5a-d as colorless oils. 
<smiles>O=C(O)C1C=CC(c2ccccc2)C(O)C1OCc1ccccc1</smiles>

Compound 5a. 92\%; $R_{f} 0.66$ (25\% EtOAc/hexanes); ${ }^{1} \mathrm{H}$ NMR $\left(\mathrm{CDCl}_{3}\right): \delta 7.48-7.30(\mathrm{~m}, 20 \mathrm{H}), 5.99$ $(\mathrm{dd}, J=10.1,2.0 \mathrm{~Hz}, 1 \mathrm{H}), 5.85(\mathrm{ddd}, J=10.1,4.3,1.9 \mathrm{~Hz}, 1 \mathrm{H}), 4.86-4.68(\mathrm{~m}, 6 \mathrm{H}), 4.25(\mathrm{br} \mathrm{s}, 1 \mathrm{H})$, 3.91 (br s, $3 \mathrm{H}) ;{ }^{13} \mathrm{C} \mathrm{NMR}\left(\mathrm{CDCl}_{3}\right): \delta 138.9,138.8,138.7,130.4,129.0,128.5,128.3,128.3,128.0$, 127.8, 127.7, 127.5, 127.4, 127.0, 79.9, 79.8, 79.1, 74.6, 72.8, 72.4, 45.8; HRMS m/z (EI) calcd for $\mathrm{C}_{33} \mathrm{H}_{33} \mathrm{O}_{3}(\mathrm{M}+\mathrm{H})^{+}$477.2430, found 477.2423.<smiles>COc1ccc([C@H]2C=C[C@@H](Cc3ccccc3)[C@H](OCc3ccccc3)[C@H]2OCc2ccccc2)cc1</smiles>

Compound 5b. 89\%; $R_{f} 0.63$ (25\% EtOAc/hexanes); ${ }^{1} \mathrm{H}$ NMR $\left(\mathrm{CDCl}_{3}\right): \delta 7.42-7.28(\mathrm{~m}, 15 \mathrm{H}), 7.24$ $(\mathrm{d}, J=8.7 \mathrm{~Hz}, 2 \mathrm{H}), 6.90(\mathrm{~d}, J=8.7 \mathrm{~Hz}, 2 \mathrm{H}), 5.97(\mathrm{ddd}, J=9.9,2.4,0.9 \mathrm{~Hz}, 1 \mathrm{H}), 5.78(\mathrm{ddd}, J=9.9$, 4.5, $1.7 \mathrm{~Hz}, 1 \mathrm{H}), 4.81-4.64(\mathrm{~m}, 6 \mathrm{H}), 4.19(\mathrm{~m}, 1 \mathrm{H}), 3.83(\mathrm{~s}, 3 \mathrm{H}), 3.80(\mathrm{~m}, 3 \mathrm{H}) ;{ }^{13} \mathrm{C} \mathrm{NMR}\left(\mathrm{CDCl}_{3}\right)$ : $\delta 158.7,139.0,138.9,138.8,131.3,130.6,129.3,128.5,128.4,128.0,127.9,127.8,127.5,113.5$, 80.2, 80.0, 79.3, 74.7, 72.9, 72.4, 55.3, 45.1; HRMS $m / z$ (EI) calcd for $\mathrm{C}_{34} \mathrm{H}_{34} \mathrm{O}_{4}(\mathrm{M})^{+}$506.2457, found 506.2446 .

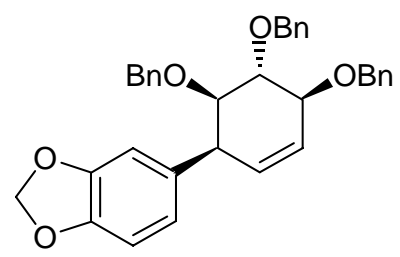

Compound 5c. 94\%; $R_{f} 0.59$ (25\% EtOAc/hexanes); ${ }^{1} \mathrm{H}$ NMR $\left(\mathrm{CDCl}_{3}\right): \delta 7.41-7.28(\mathrm{~m}, 15 \mathrm{H}), 6.81$ (m, 1H), 6.78 (br s, 1H), 6.75 (dd, $J=8.1,1.5 \mathrm{~Hz}, 1 \mathrm{H}), 5.96(\mathrm{~s}, 2 \mathrm{H}), 5.88$ (ddd, $J=10.1,2.4,1.3$ $\mathrm{Hz}, 1 \mathrm{H}), 5.73$ (ddd, $J=10.1,4.7,1.9 \mathrm{~Hz}, 1 \mathrm{H}), 4.79-4.64(\mathrm{~m}, 6 \mathrm{H}), 4.16(\mathrm{~m}, 1 \mathrm{H}), 3.81(\mathrm{~m}, 2 \mathrm{H}), 3.73$ $(\psi \mathrm{t}, J=4.5 \mathrm{~Hz}, 1 \mathrm{H}) ;{ }^{13} \mathrm{C} \mathrm{NMR}\left(\mathrm{CDCl}_{3}\right): \delta 147.6,146.7,139.0,138.8,132.5,129.1,128.6,128.4$, 128.1, 128.0, 127.8, 127.7, 127.6, 123.5, 110.9, 107.9, 101.1, 80.1, 80.0, 79.2, 74.8, 73.1, 72.5, 45.6; HRMS $m / z$ (EI) calcd for $\mathrm{C}_{34} \mathrm{H}_{32} \mathrm{O}_{5}(\mathrm{M})^{+} 520.2250$, found 520.2265. 
<smiles>COc1cc([C@H]2C=C[C@@H](OCc3ccccc3)[C@H](OCc3ccccc3)[C@H]2O)cc2c1OCO2</smiles>

Compound 5d. 96\%; $R_{f} 0.57$ (25\% EtOAc/hexanes); ${ }^{1} \mathrm{H}$ NMR $\left(\mathrm{CDCl}_{3}\right): \delta 7.42-7.31(\mathrm{~m}, 15 \mathrm{H}), 6.54$ $(\mathrm{d}, J=1.4 \mathrm{~Hz}, 1 \mathrm{H}), 6.47(\mathrm{~d}, J=1.4 \mathrm{~Hz}, 1 \mathrm{H}), 5.98(\mathrm{~s}, 2 \mathrm{H}), 5.93(\mathrm{ddd}, J=9.9,2.8,1.1 \mathrm{~Hz}, 1 \mathrm{H}), 5.78$ (ddd, $J=9.9,4.7,1.7 \mathrm{~Hz}, 1 \mathrm{H}), 4.80-4.68(\mathrm{~m}, 6 \mathrm{H}), 4.18(\mathrm{~m}, 1 \mathrm{H}), 3.85(\mathrm{~m}, 2 \mathrm{H}), 3.83(\mathrm{~s}, 3 \mathrm{H}), 3.73$ $(\psi \mathrm{t}, J=4.7 \mathrm{~Hz}, 1 \mathrm{H}) ;{ }^{13} \mathrm{C} \mathrm{NMR}\left(\mathrm{CDCl}_{3}\right): \delta 148.6,143.1,138.9,138.8,134.3,133.2,129.1,128.5$, 128.4, 128.0, 127.9, 127.7, 127.6, 127.5, 110.0, 104.4, 101.5, 79.9, 79.8, 79.3, 74.6, 73.2, 72.2, 56.6, 45.7; HRMS $m / z$, EI) calcd for $\mathrm{C}_{35} \mathrm{H}_{34} \mathrm{O}_{6}(\mathrm{M})^{+}$550.2355, found 550.2356.<smiles>O[C@H]1C=C[C@H](c2ccc3c(c2)OCO3)[C@H](O)[C@@H]1O</smiles>

Compound 10c. Ammonia gas was liquified at $-78{ }^{\circ} \mathrm{C}$ in two flasks $(\sim 10 \mathrm{~mL}$ in each). The first flask was charged with $\mathrm{Li}(0.026 \mathrm{~g}, 3.7 \mathrm{mmol})$, while to the second flask was added compound $\mathbf{5 c}$ $(0.16 \mathrm{~g}, 0.31 \mathrm{mmol})$ in THF $(5 \mathrm{~mL})$. After the complete dissolution of Li the deep-blue solution was added via cannula to the solution of $\mathbf{5 c}$ until the blue color persisted for more than $30 \mathrm{~s}$. The reaction mixture was quenched with dry $\mathrm{NH}_{4} \mathrm{Cl}(0.2 \mathrm{~g}, 3.7 \mathrm{mmol})$ and allowed to stand at $\mathrm{rt}$ until the complete evaporation of $\mathrm{NH}_{3}$. The residue was diluted with water $(20 \mathrm{~mL})$ and extracted with EtOAc $(3 \times 30 \mathrm{~mL})$. After the removal of the solvent under reduced pressure the residue was recrystallized from acetone to give 10c as white needles $(0.047 \mathrm{~g}$, yield $61 \%)$; mp $112-113{ }^{\circ} \mathrm{C}$; $R_{f} 0.39\left(10 \% \mathrm{MeOH} / \mathrm{CH}_{2} \mathrm{Cl}_{2}\right) ;{ }^{1} \mathrm{H}$ NMR $\left(\mathrm{D}_{2} \mathrm{O}\right): \delta 6.90(\mathrm{~d}, J=8.0 \mathrm{~Hz}, 1 \mathrm{H}), 6.83(\mathrm{br} \mathrm{s}, 1 \mathrm{H}), 6.79(\mathrm{br}$ $\mathrm{d}, J=8.0 \mathrm{~Hz}, 1 \mathrm{H}), 5.98(\mathrm{~s}, 2 \mathrm{H}), 5.85-5.75(\mathrm{~m}, 2 \mathrm{H}), 4.21(\mathrm{br} \mathrm{d}, J=7.4 \mathrm{~Hz}, 1 \mathrm{H}), 3.94$ (dd, $J=10.7$, $6.3 \mathrm{~Hz}, 1 \mathrm{H}), 3.79(\psi \mathrm{t}, J=4.3 \mathrm{~Hz}, 1 \mathrm{H}), 3.57(\mathrm{dd}, J=10.7,7.7 \mathrm{~Hz}, 1 \mathrm{H}) ;{ }^{13} \mathrm{C}$ NMR $\left(\mathrm{D}_{2} \mathrm{O}\right): \delta 147.2$, $146.4,131.9,129.1,128.8,123.9,110.9,108.3,101.2,72.9,72.7,70.7,47.2 ; \mathrm{HRMS} m / z$ (EI) calcd for $\mathrm{C}_{13} \mathrm{H}_{14} \mathrm{O}_{5}(\mathrm{M})^{+} 250.0841$, found 250.0840 .<smiles>CC1(C)O[C@H]2[C@H](O)[C@H](O)[C@H](O)[C@@H](c3ccc4c(c3)OCO4)[C@H]2O1</smiles>

Compound 9c. To the solution of $\mathbf{5 c}(0.114 \mathrm{~g}, 0.22 \mathrm{mmol})$ in $5 \mathrm{~mL}$ of mixture acetone- $\mathrm{H}_{2} \mathrm{O}(9: 1)$ was added NMO $(0.036 \mathrm{~g}, 0.26 \mathrm{mmol})$ and $2.5 \% \mathrm{OsO}_{4}$ in $t$-butanol $(0.224 \mathrm{~g}$ solution, $0.022 \mathrm{mmol})$. The mixture was stirred overnight, then quenched with $10 \% \mathrm{NaHSO}_{3}(1 \mathrm{~g})$, diluted with water $(20$ $\mathrm{mL})$, extracted with ether $(3 \times 20 \mathrm{~mL})$ and dried $\left(\mathrm{MgSO}_{4}\right)$. After the removal of the solvent the crude diol was dissolved in DMF (5 mL). 2,2-Dimethoxypropane $(0.046 \mathrm{~g}, 0.44 \mathrm{mmol})$ and $\mathrm{TsOH}$ 
$(0.0023 \mathrm{~g}, 0.022 \mathrm{mmol})$ were added and the reaction mixture was stirred for $3 \mathrm{~h}$ at $\mathrm{rt}$. The mixture was diluted with water $(30 \mathrm{~mL})$, extracted with ether $(3 \times 30 \mathrm{~mL})$ and dried $\left(\mathrm{MgSO}_{4}\right)$. After the removal of the solvent the residue was chromatographed (25\% EtOAc/hexanes). The pure acetonide $(0.090 \mathrm{~g})$ was dissolved in $\mathrm{MeOH}(10 \mathrm{~mL})$ and hydrogenolyzed overnight $\left(50 \mathrm{psi}_{2}, 10 \% \mathrm{Pd} / \mathrm{C}\right)$. The resulting solution was filtered through a layer of celite and the solvent was removed to give pure 9c $(0.049 \mathrm{~g}, 69 \%$ from $5 \mathrm{c}) ; 300 \mathrm{MHz}{ }^{1} \mathrm{H}$ NMR $\left(\mathrm{CDCl}_{3}\right): \delta 6.82(\mathrm{~d}, J=1.4 \mathrm{~Hz}, 1 \mathrm{H}), 6.79(\mathrm{~d}, J=$ $8.0 \mathrm{~Hz}, 1 \mathrm{H}), 6.73(\mathrm{dd}, J=8.0,1.4 \mathrm{~Hz}, 1 \mathrm{H}), 5.93(\mathrm{~s}, 2 \mathrm{H}), 4.58(\mathrm{dd}, J=6.6,6.1 \mathrm{~Hz}, 1 \mathrm{H}), 4.31(\mathrm{dd}, J=$ $6.9,6.1 \mathrm{~Hz}, 1 \mathrm{H}), 3.94(\mathrm{dd}, J=5.5,3.9 \mathrm{~Hz}, 1 \mathrm{H}), 3.81(\mathrm{dd}, J=8.0,6.9 \mathrm{~Hz}, 1 \mathrm{H}), 3.66(\mathrm{dd}, J=8.0,5.5$ $\mathrm{Hz}, 1 \mathrm{H}), 3.30(\mathrm{dd}, J=6.6,3.9 \mathrm{~Hz}, 1 \mathrm{H}), 1.48(\mathrm{~s}, 3 \mathrm{H}), 1.34(\mathrm{~s}, 3 \mathrm{H}) ; 300 \mathrm{MHz}{ }^{1} \mathrm{H}$ NMR $\left(\mathrm{D}_{2} \mathrm{O}, 45^{\circ} \mathrm{C}\right)$ : $\delta 7.17(\mathrm{~d}, J=1.7 \mathrm{~Hz}, 1 \mathrm{H}), 7.15(\mathrm{~d}, J=8.0 \mathrm{~Hz}, 1 \mathrm{H}), 7.09(\mathrm{dd}, J=8.0,1.7 \mathrm{~Hz}, 1 \mathrm{H}), 6.21(\mathrm{~s}, 2 \mathrm{H}), 5.02$ $(\mathrm{dd}, J=8.5,7.2 \mathrm{~Hz}, 1 \mathrm{H}), 4.58(\mathrm{dd}, J=8.5,7.2 \mathrm{~Hz}, 1 \mathrm{H}), 4.14(\mathrm{dd}, J=4.4,4.1 \mathrm{~Hz}, 1 \mathrm{H}), 4.00(\mathrm{dd}, J=$ 9.1, 8.8 Hz, 1H), $3.82(\mathrm{dd}, J=9.1,4.1 \mathrm{~Hz}, 1 \mathrm{H}), 3.44(\mathrm{dd}, J=8.8,4.4 \mathrm{~Hz}, 1 \mathrm{H}), 1.72(\mathrm{~s}, 3 \mathrm{H}), 1.62(\mathrm{~s}$, $3 \mathrm{H}) ;{ }^{13} \mathrm{C} \mathrm{NMR}\left(\mathrm{CDCl}_{3}\right): \delta 148.1,147.0,131.4,122.3,109.6,109.1,108.5,101.2,78.7,75.9,75.1$, 74.3, 74.0, 46.5, 28.0, 25.5; HRMS $m / z$ (EI) calcd for $\mathrm{C}_{16} \mathrm{H}_{20} \mathrm{O}_{7}(\mathrm{M})^{+} 324.1209$, found 324.1215.

\section{References:}

1. Tejima, S.; Ness, R. K.; Kaufman, R. L.; Fletcher, H. G., Jr. Carbohydr. Res. 1968, 7, 485.

2. Tsuda, Y.; Nunozawa, T.; Yoshimoto, K. Chem. Pharm. Bull. 1980, 28, 3223.

3. Kornienko, A.; d'Alarcao, M. Tetrahedron Lett. 1997, 38, 6497.

4. Ziegler, F. E.; Wang, Y. J. Org. Chem. 1998, 63, 7920.

5. 3,4-Methylenedioxy-1-bromobenzene was prepared according to: Gensler, W. J.; Stouffer, J. E. J. Org. Chem. 1958, 23, 908. 5-Methoxy-3,4-methylenedioxy-1-bromobenzene was made following: Comber, M. F.; Sargent, M. V. Aust. J. Chem. 1985, 38, 1481. Iinuma M.; Tanaka T.; Matsuura, S. Yakugaku Zasshi 1983, 103, 994. 


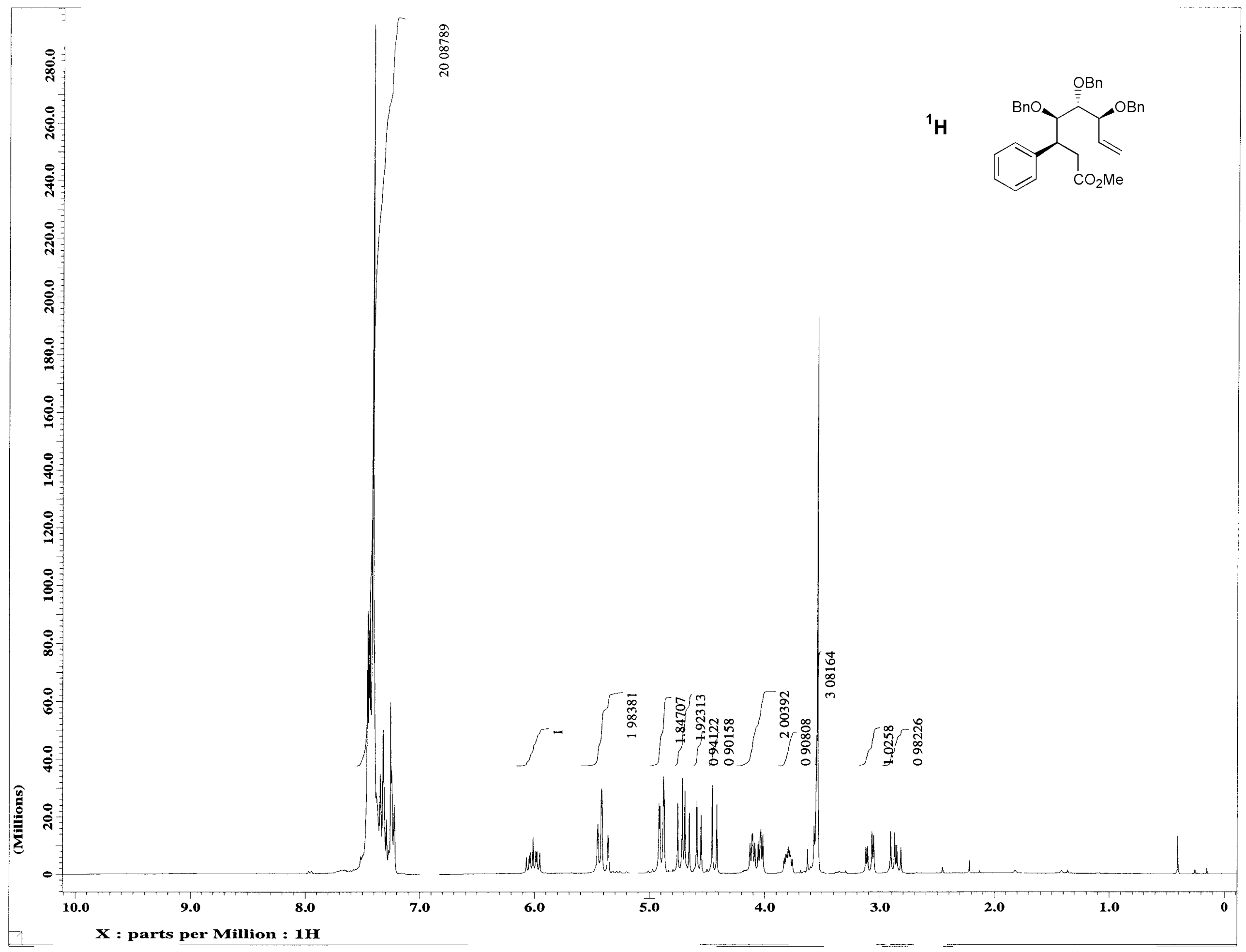




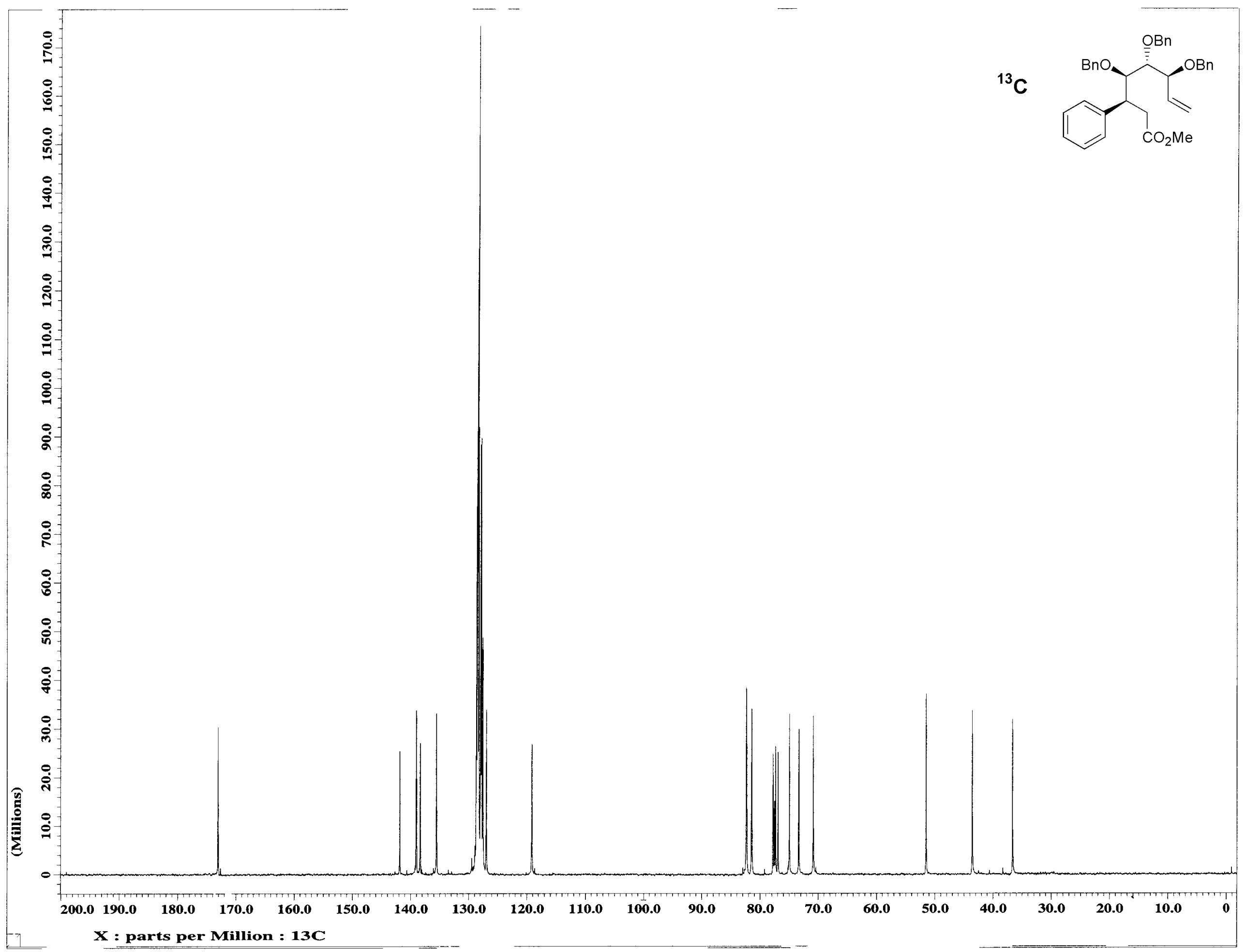


S14

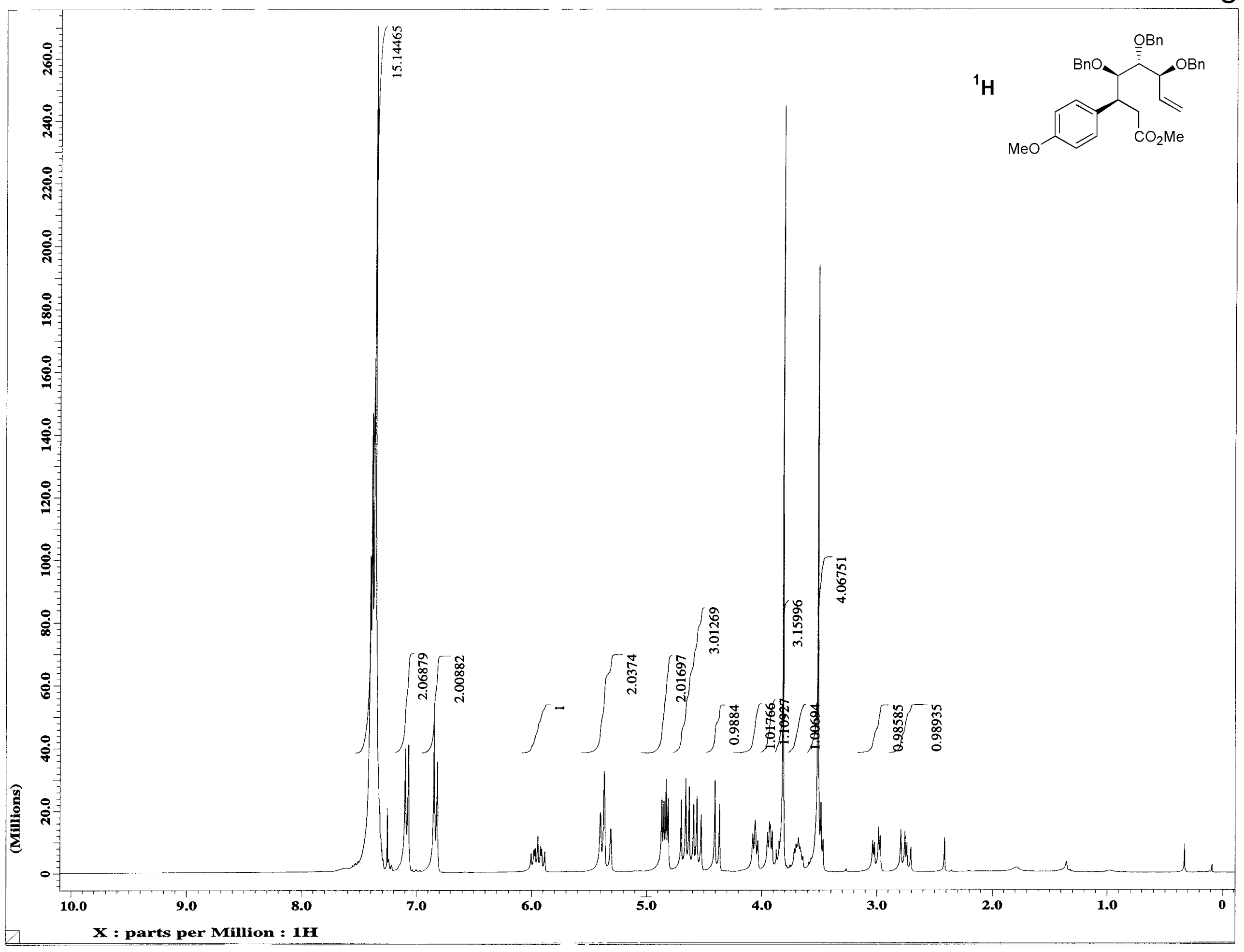




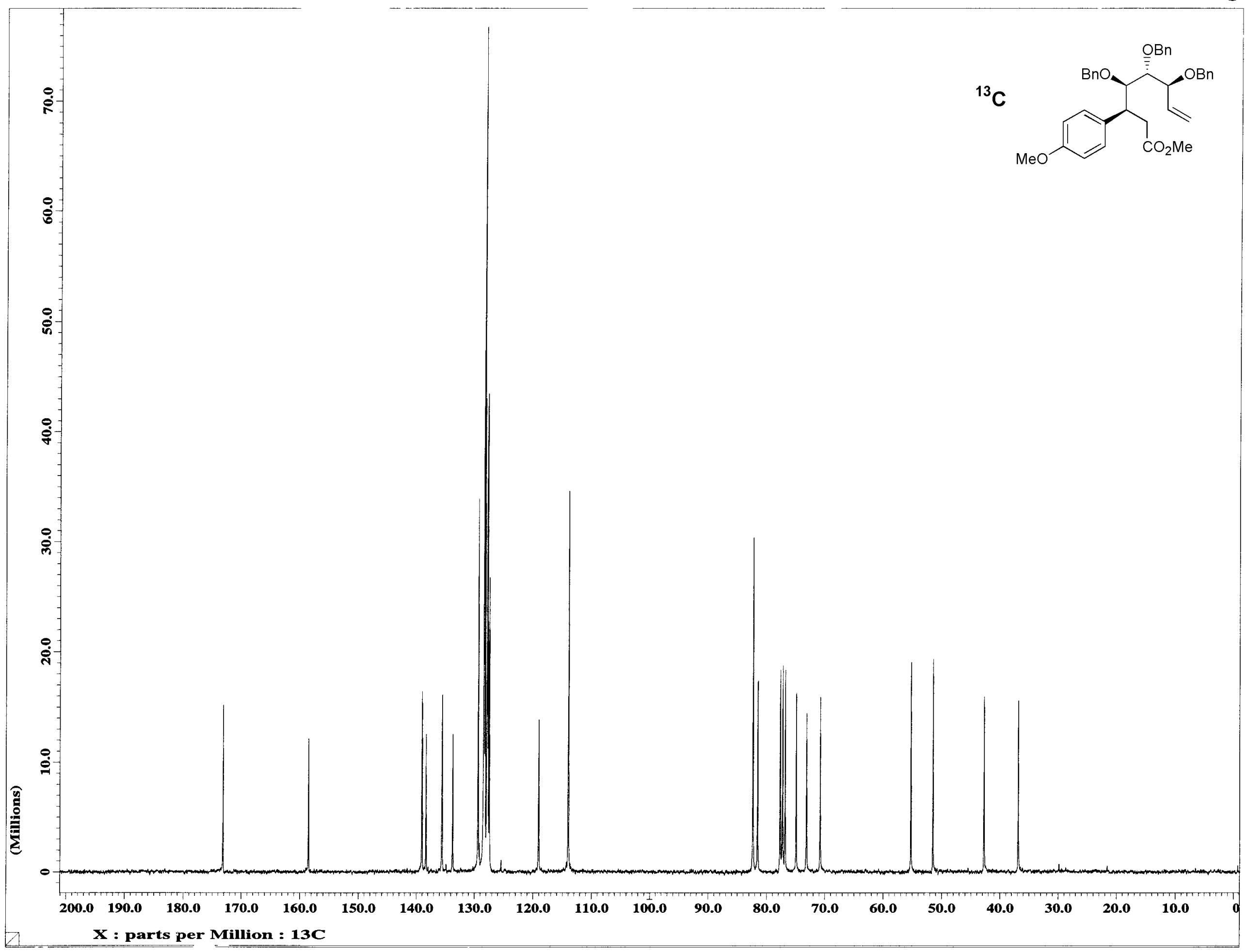




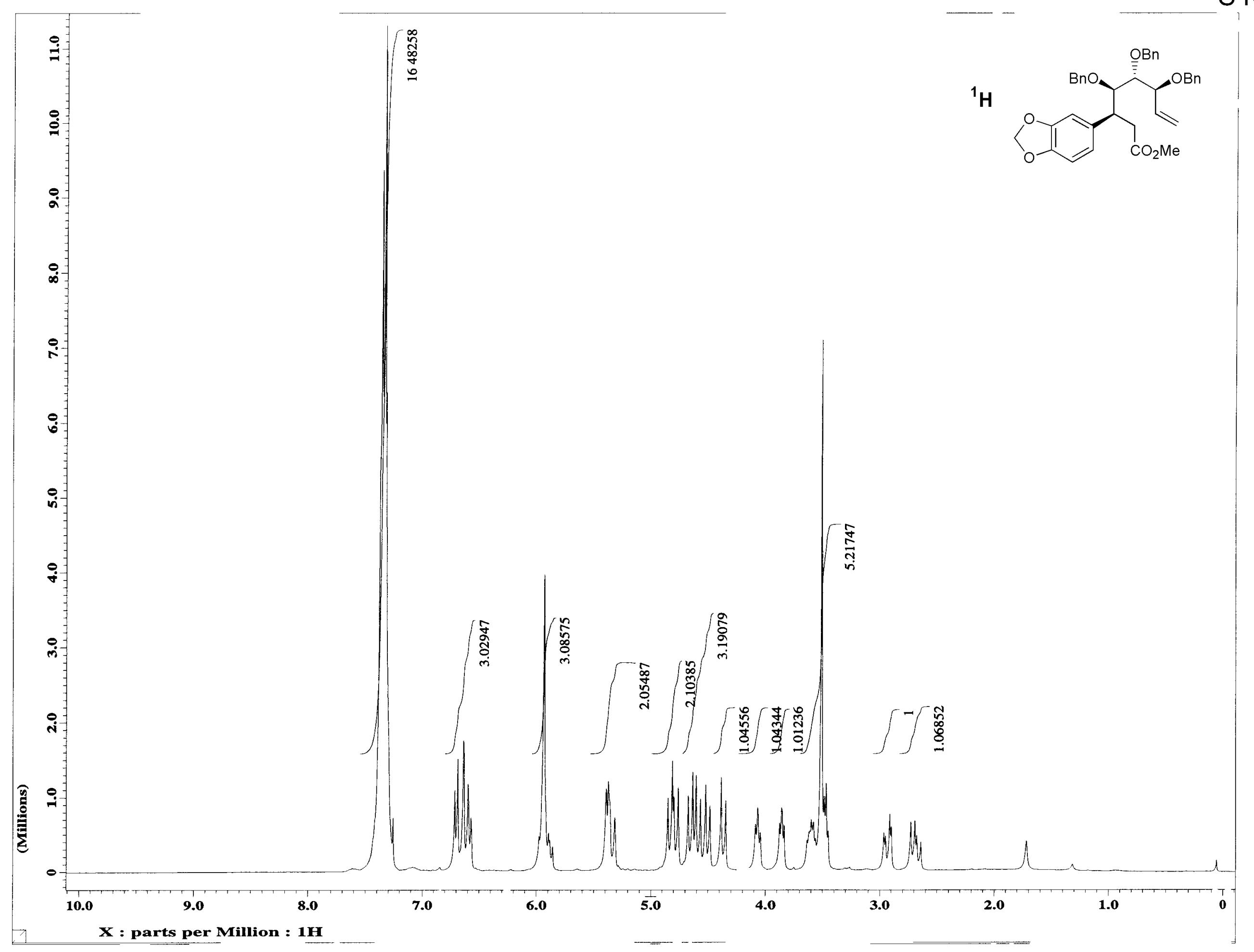




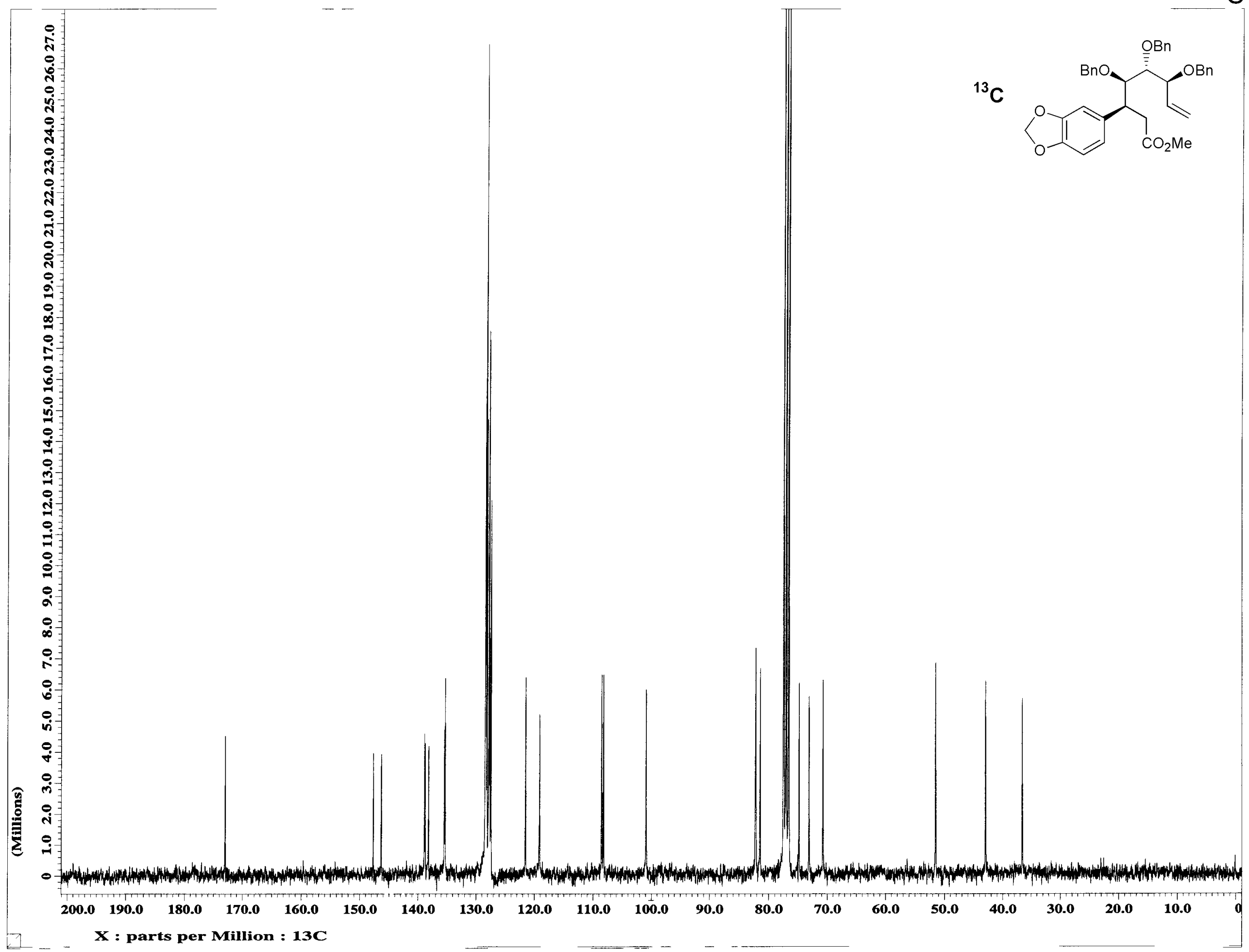


S18

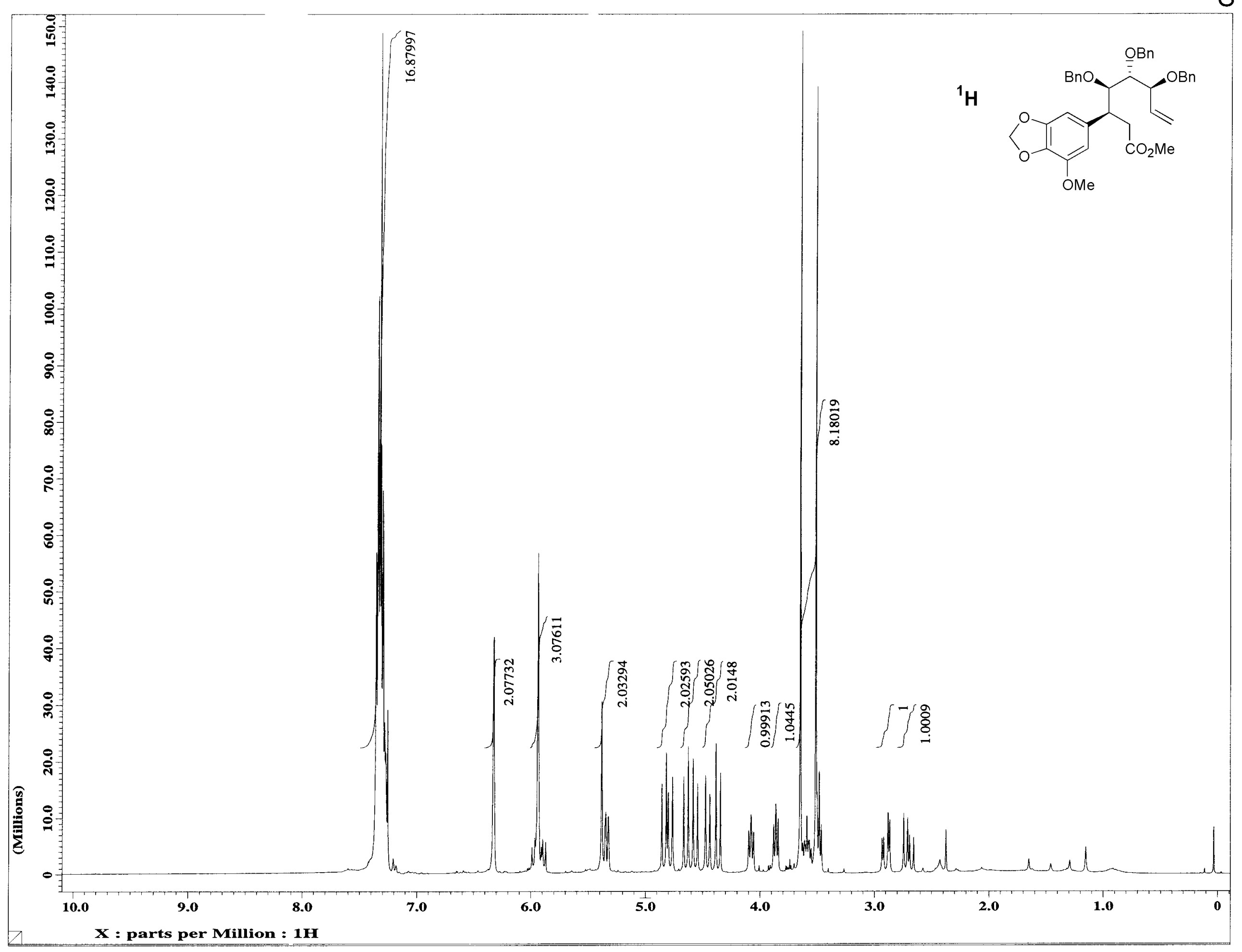




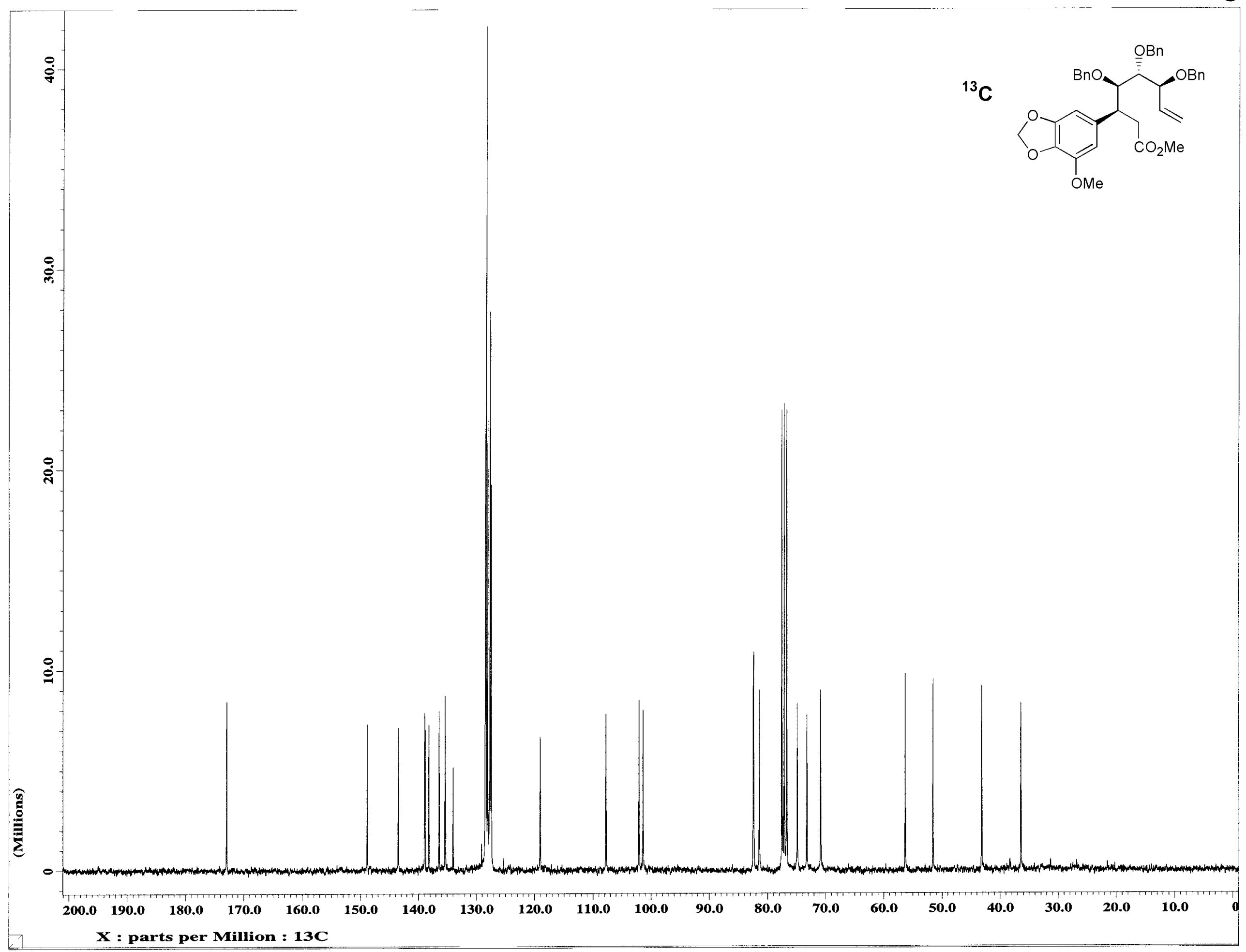




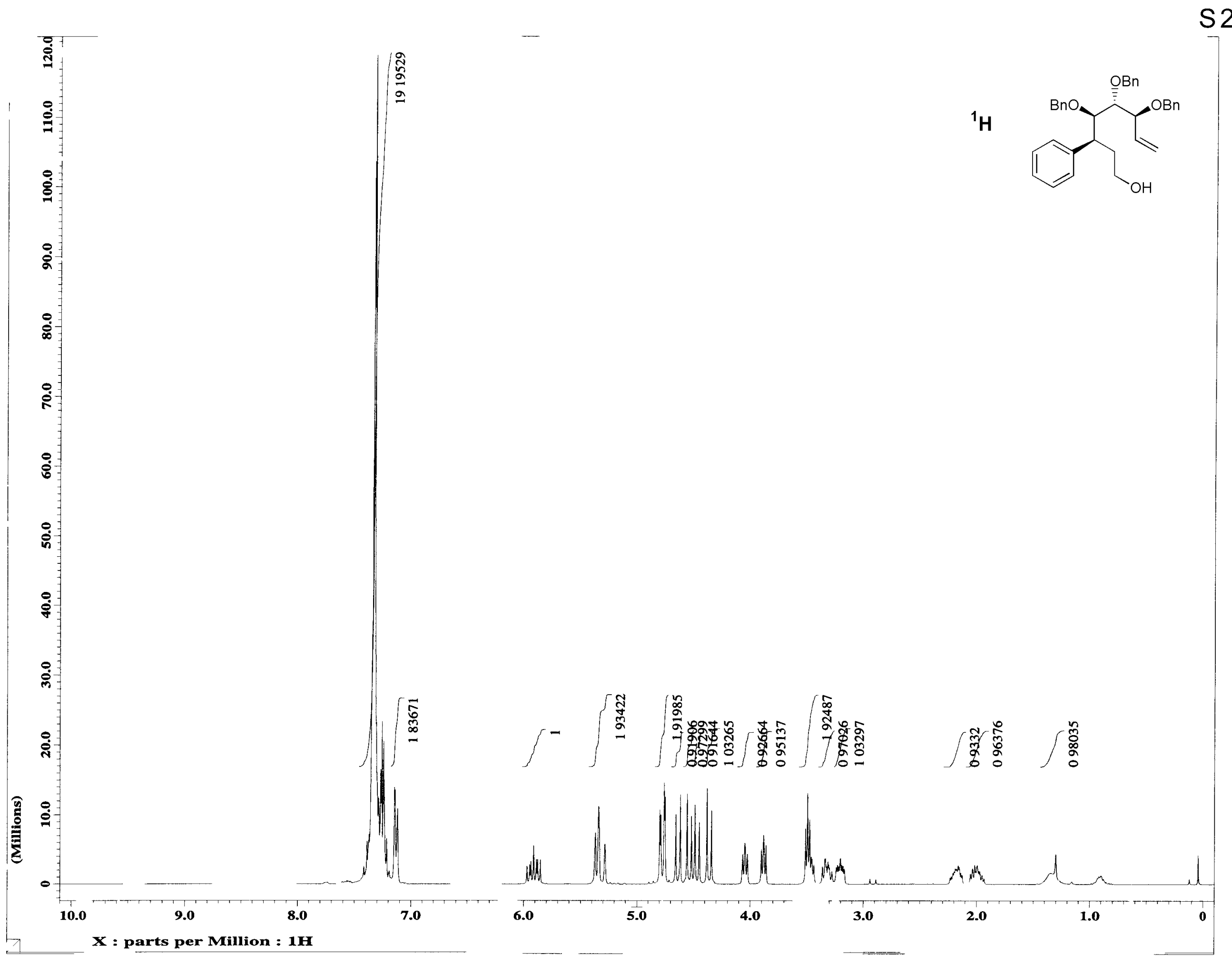




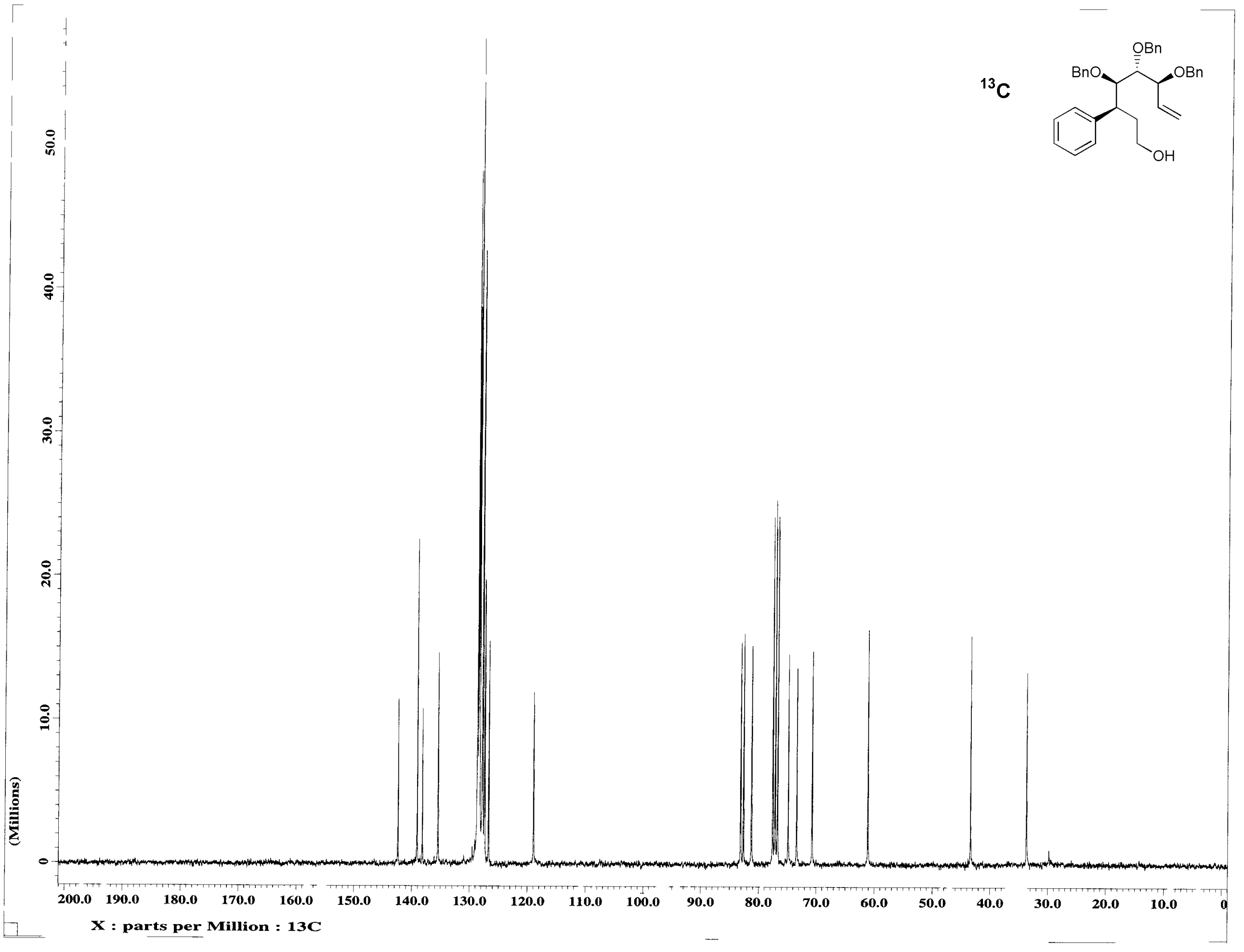




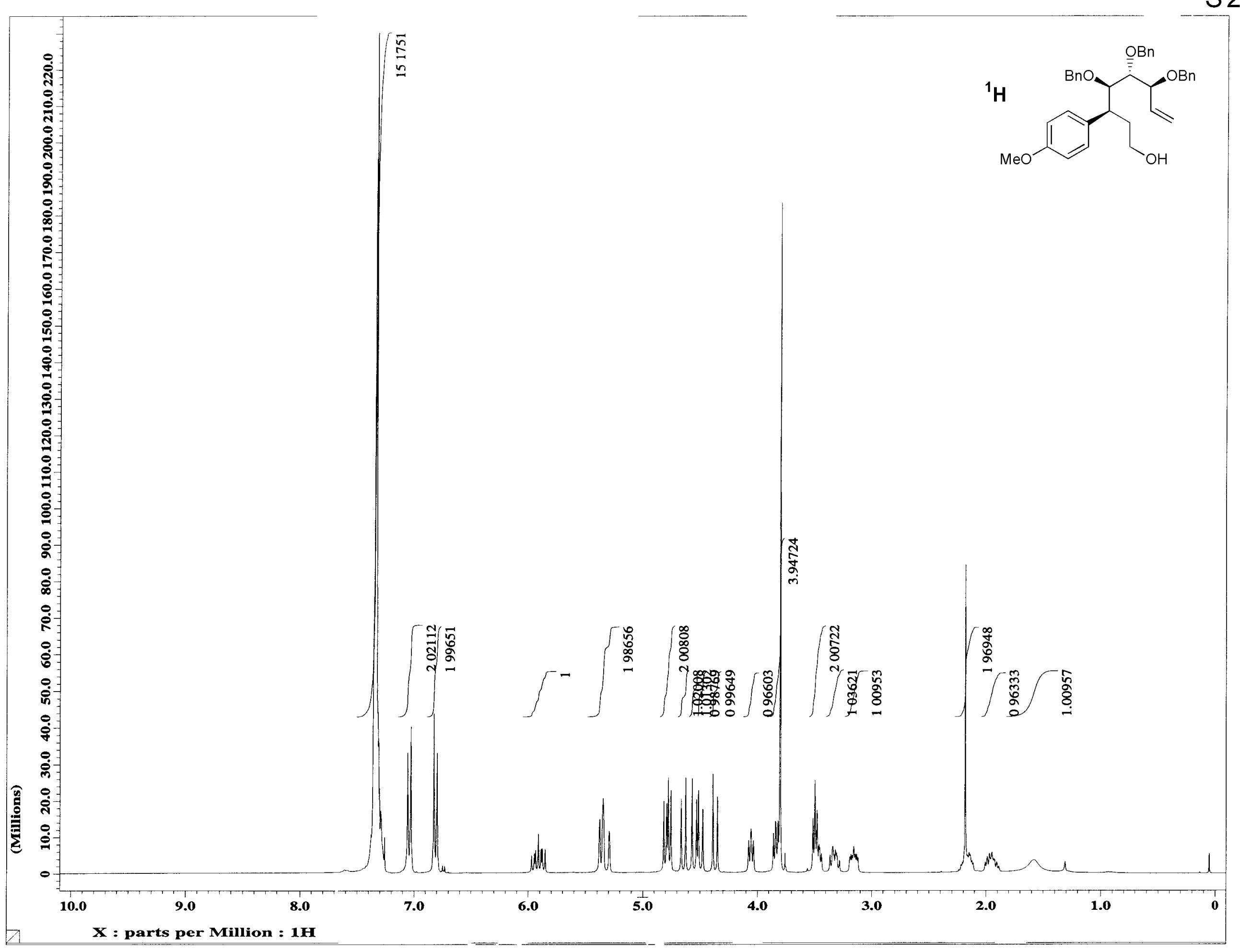




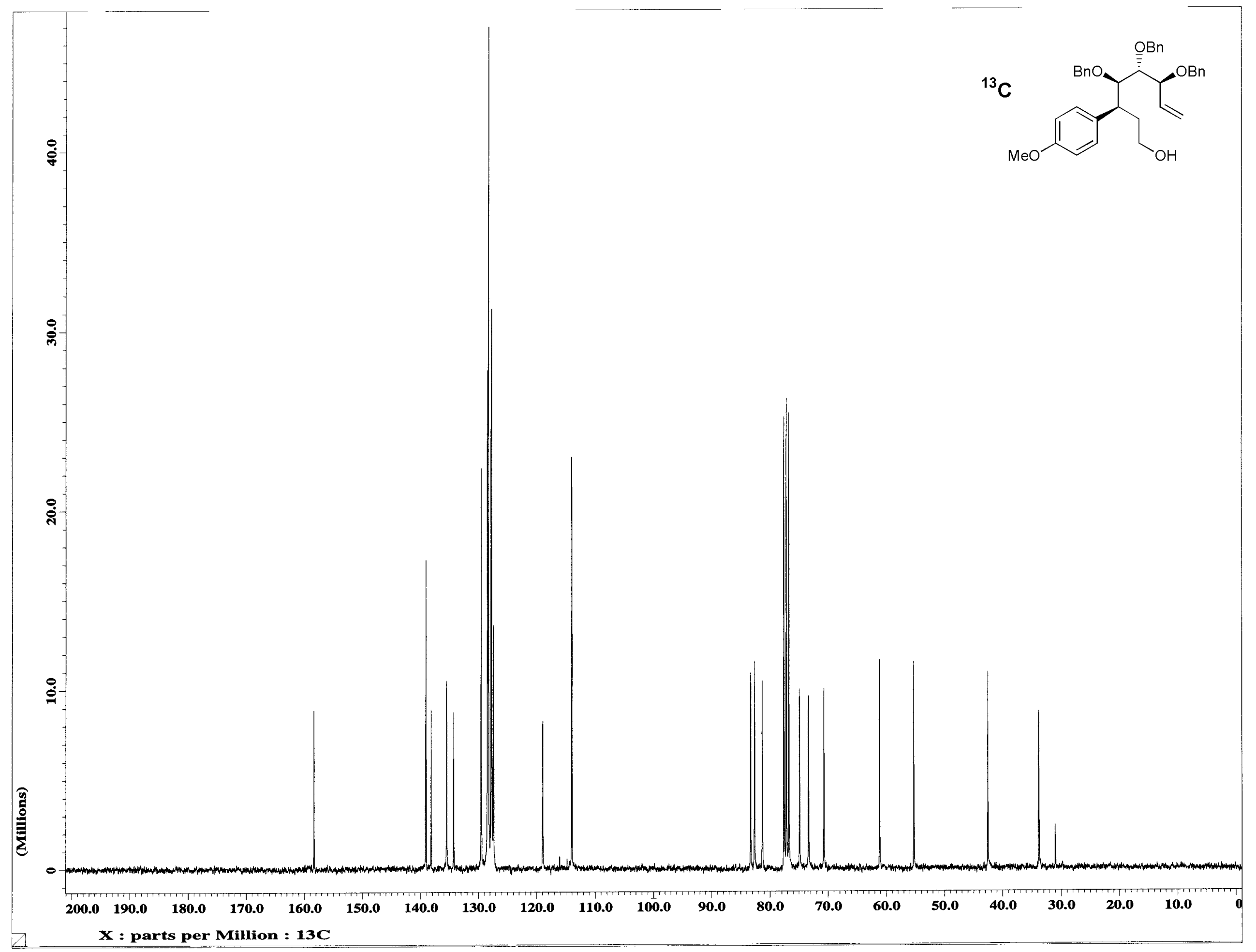




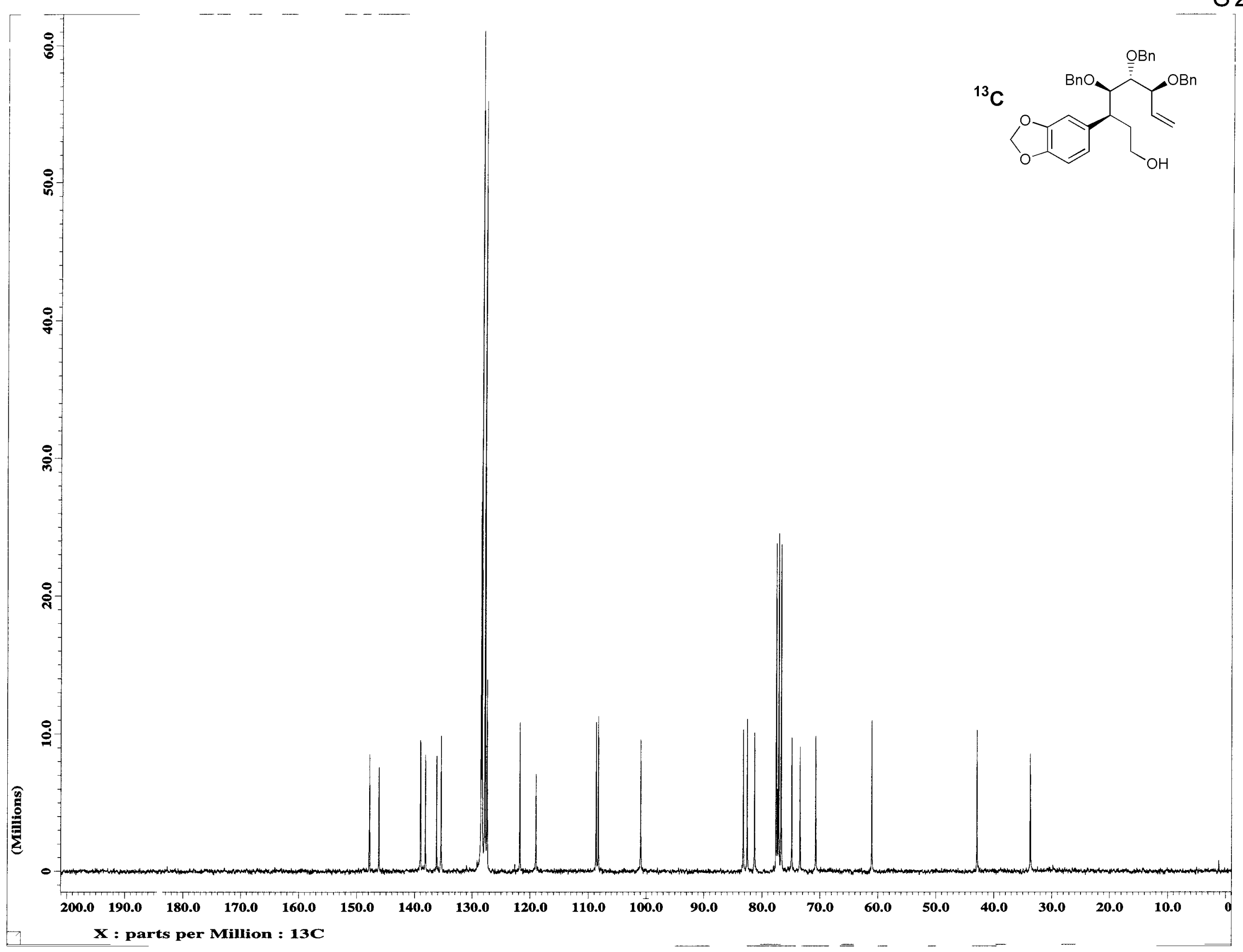




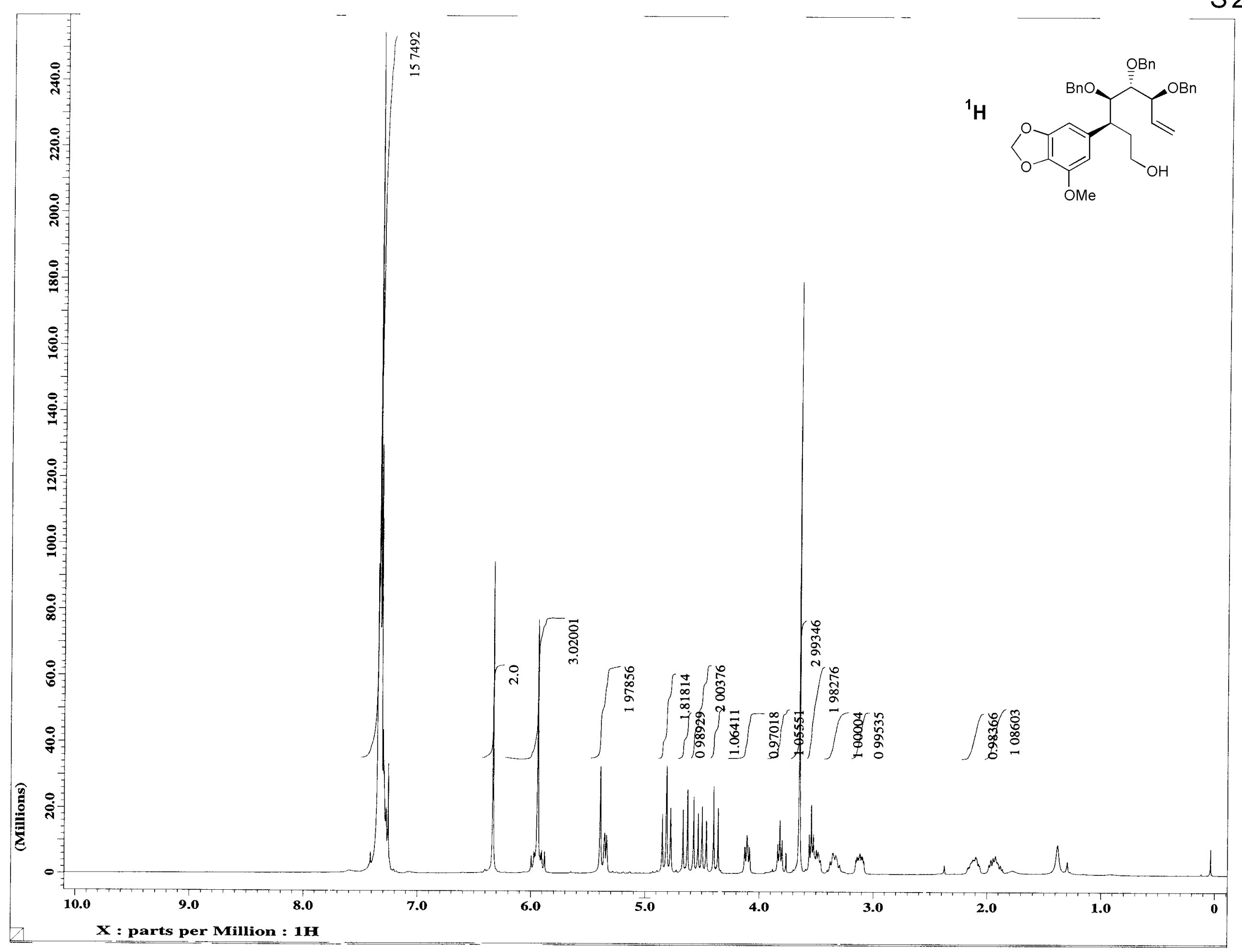




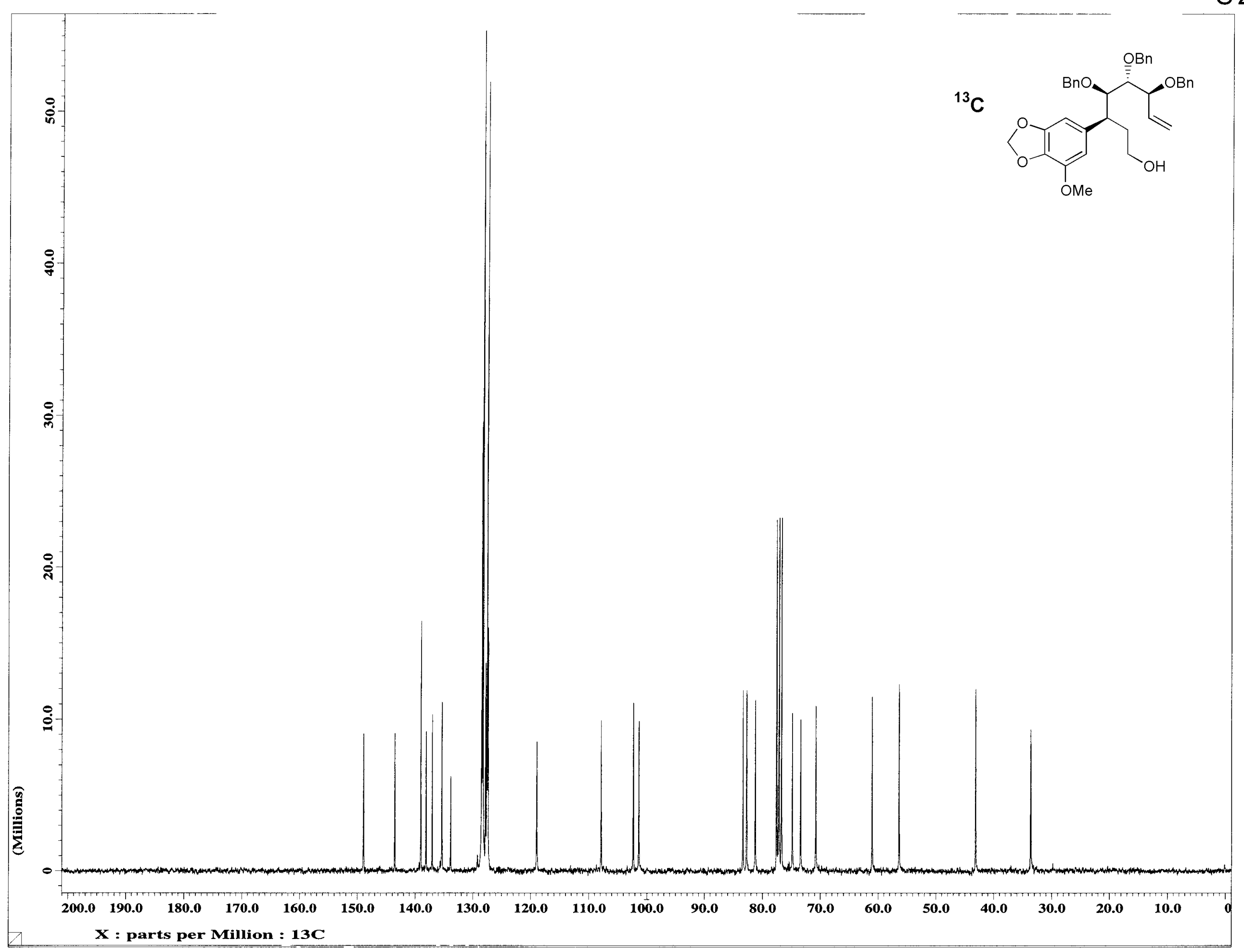




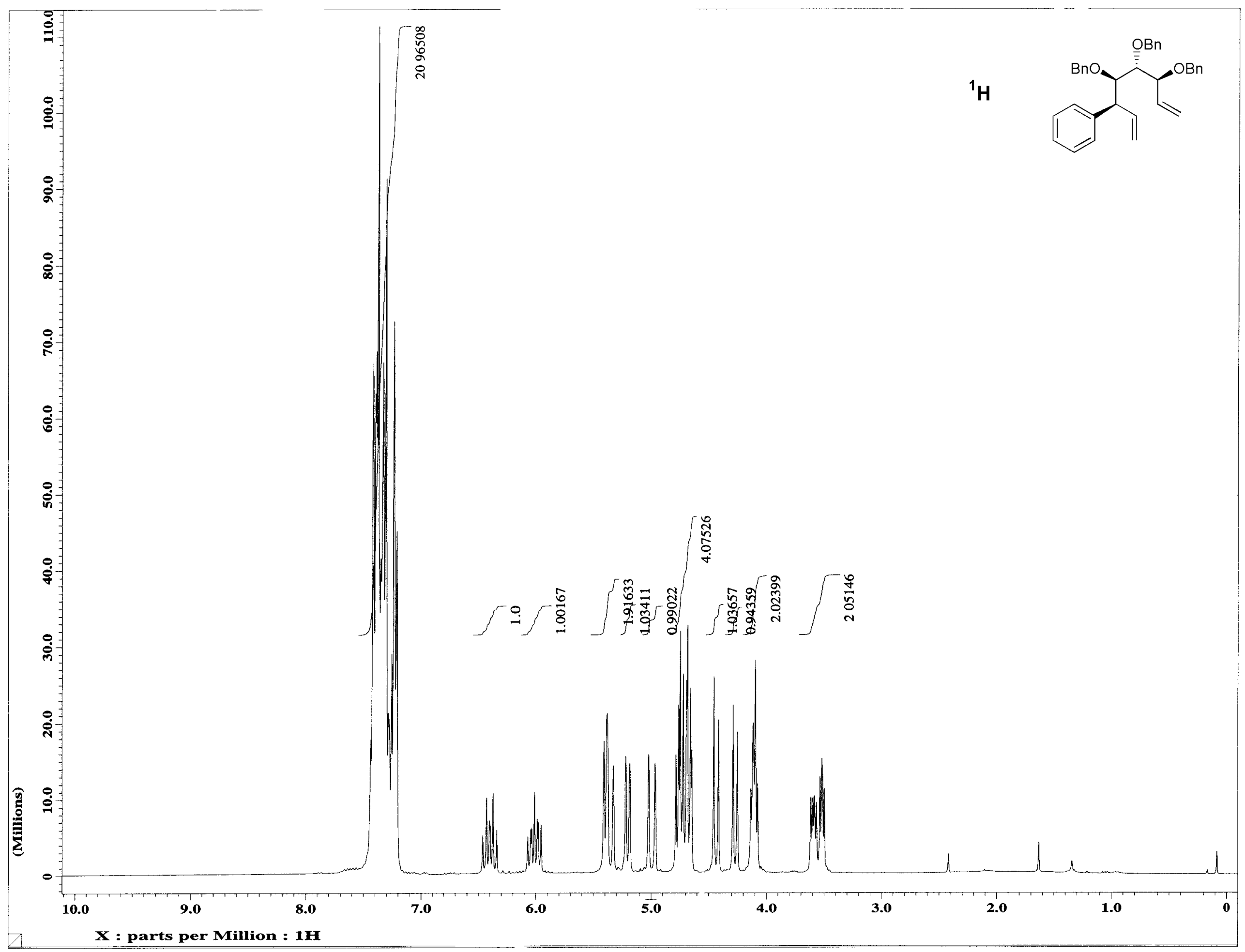




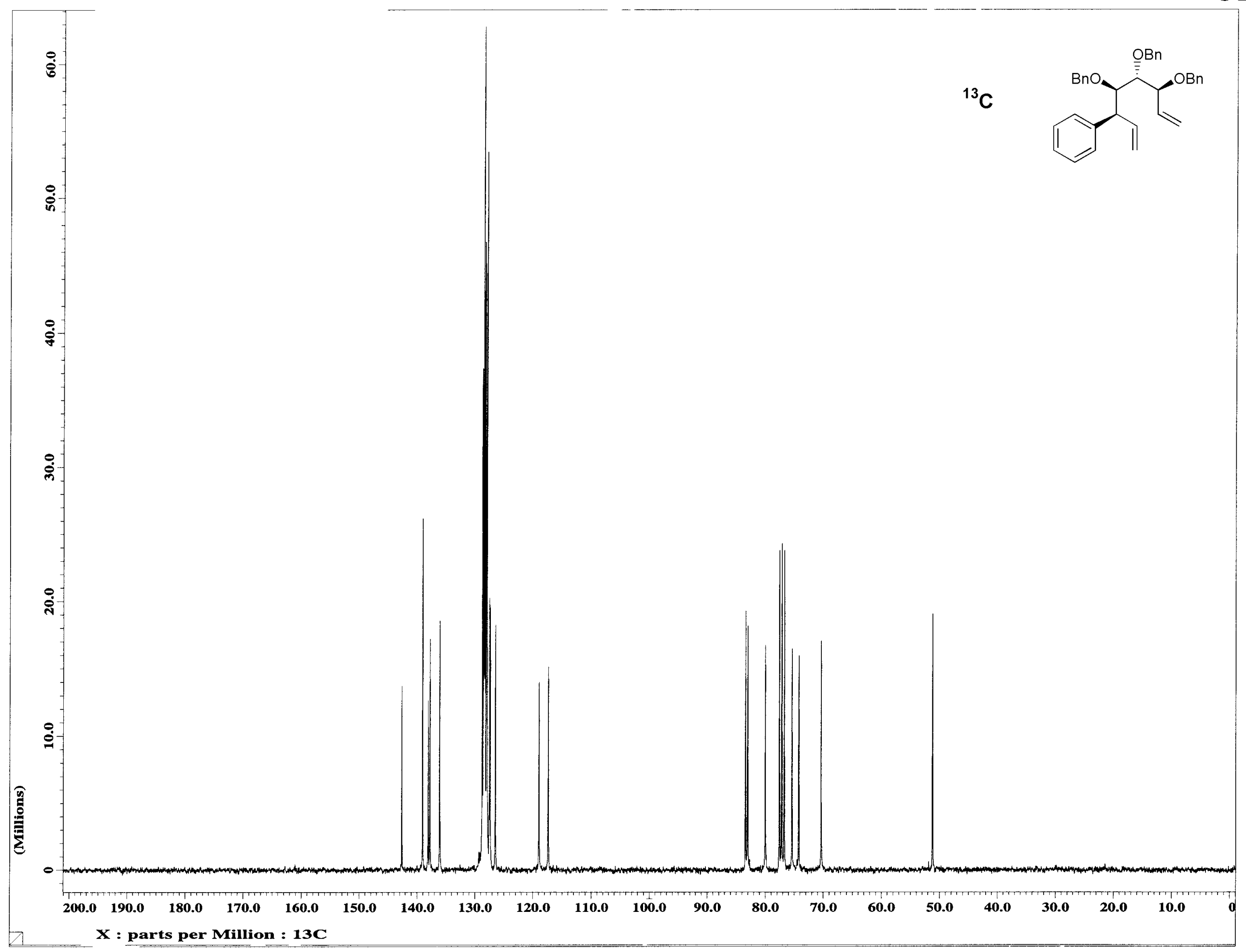




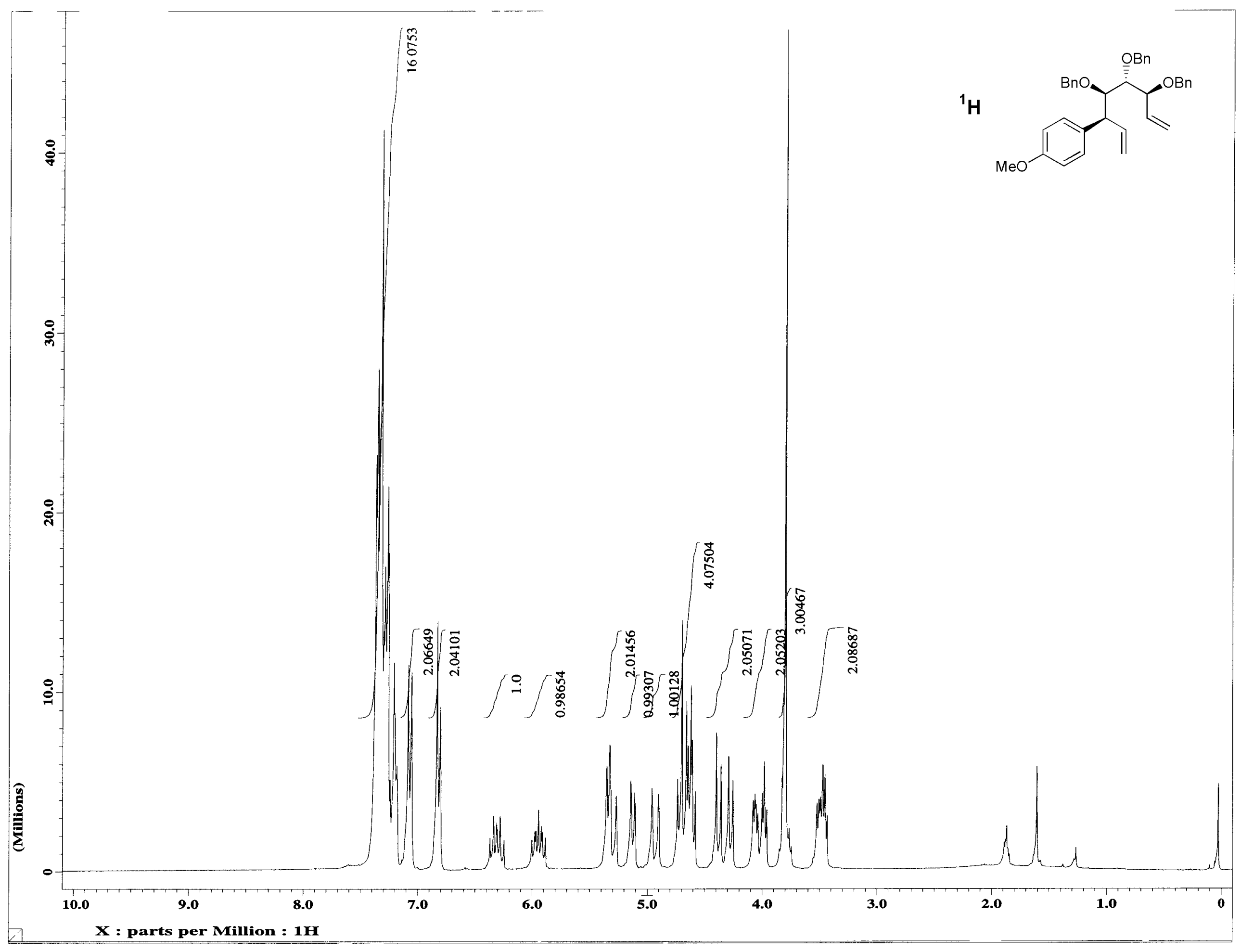




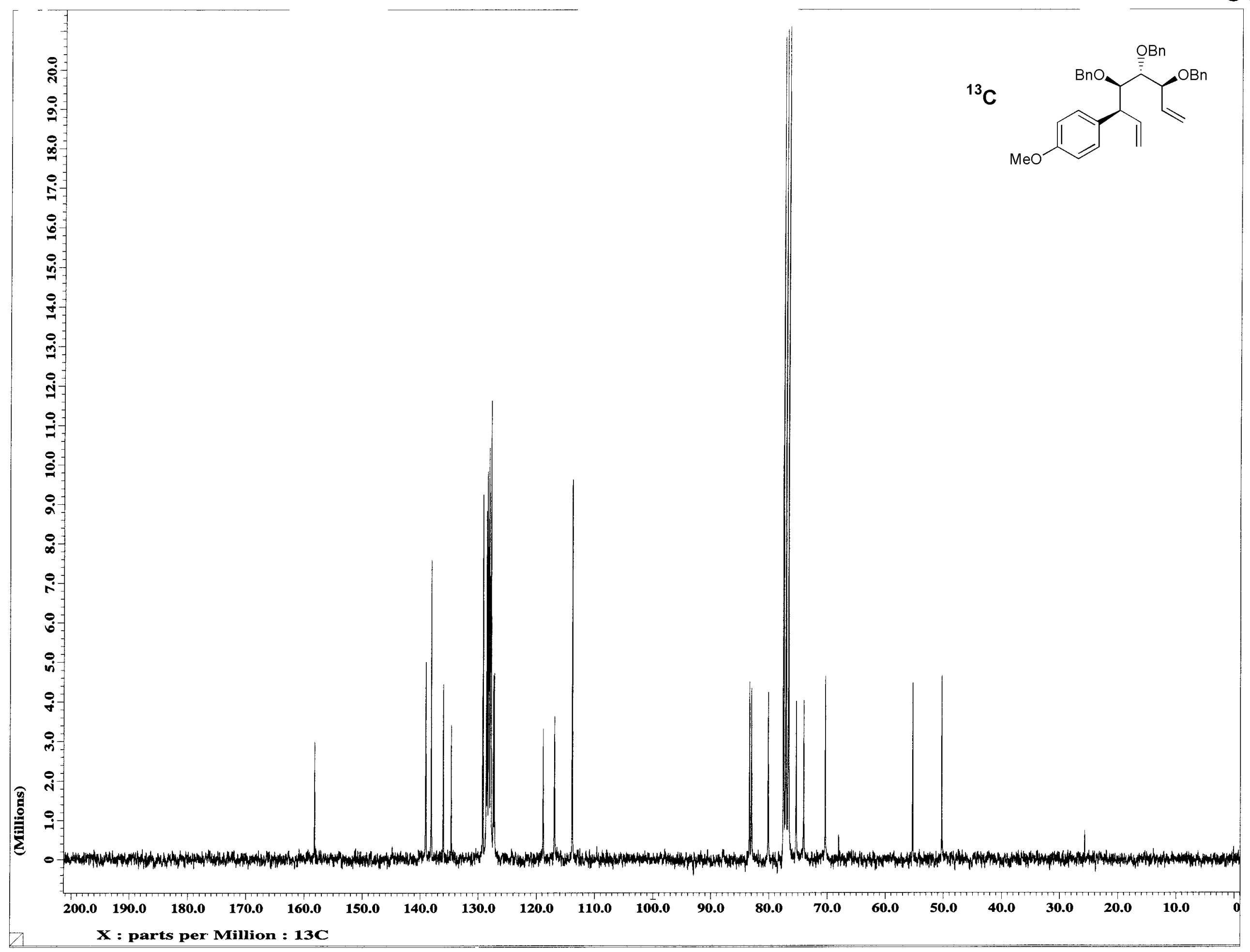




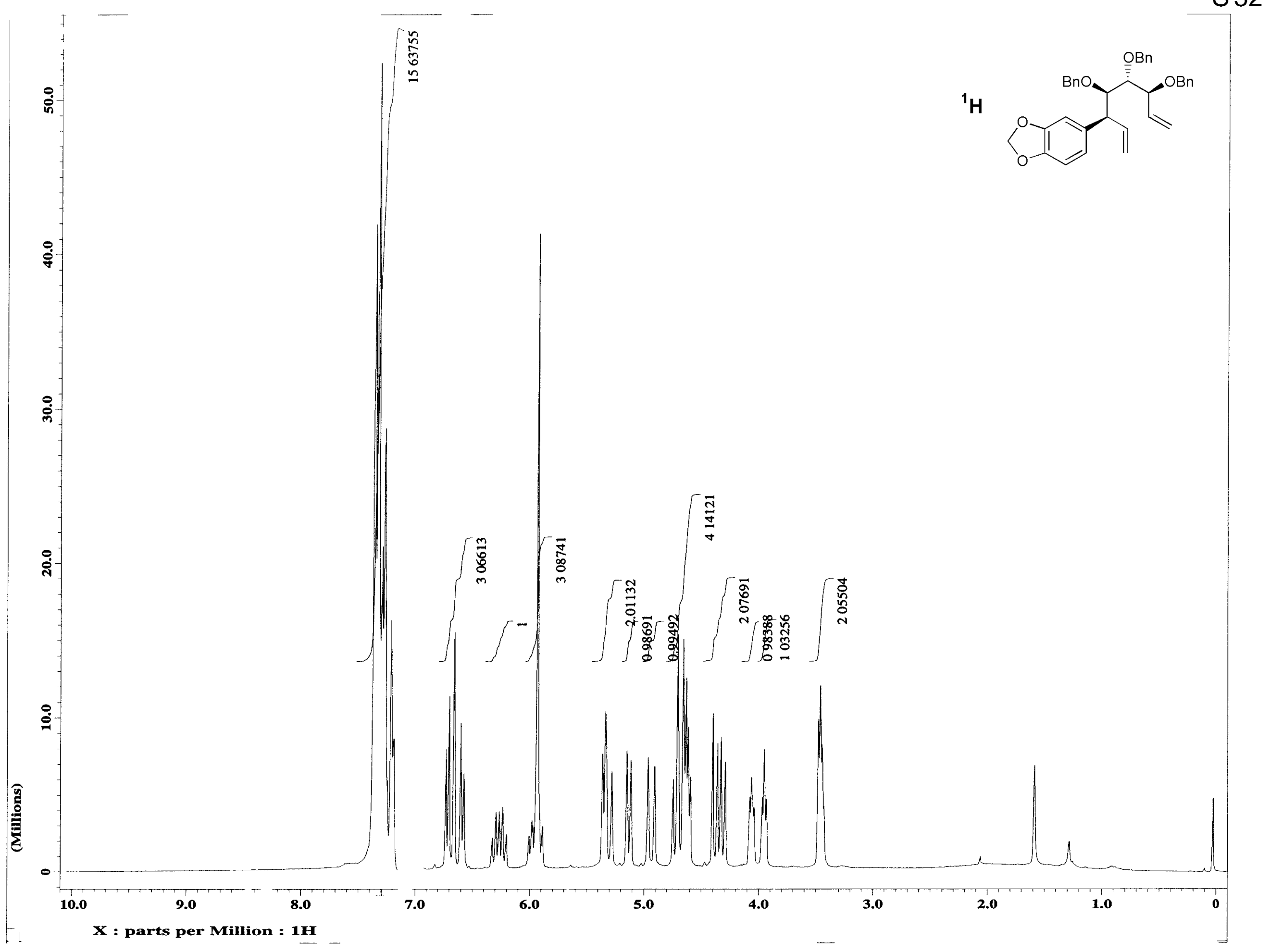




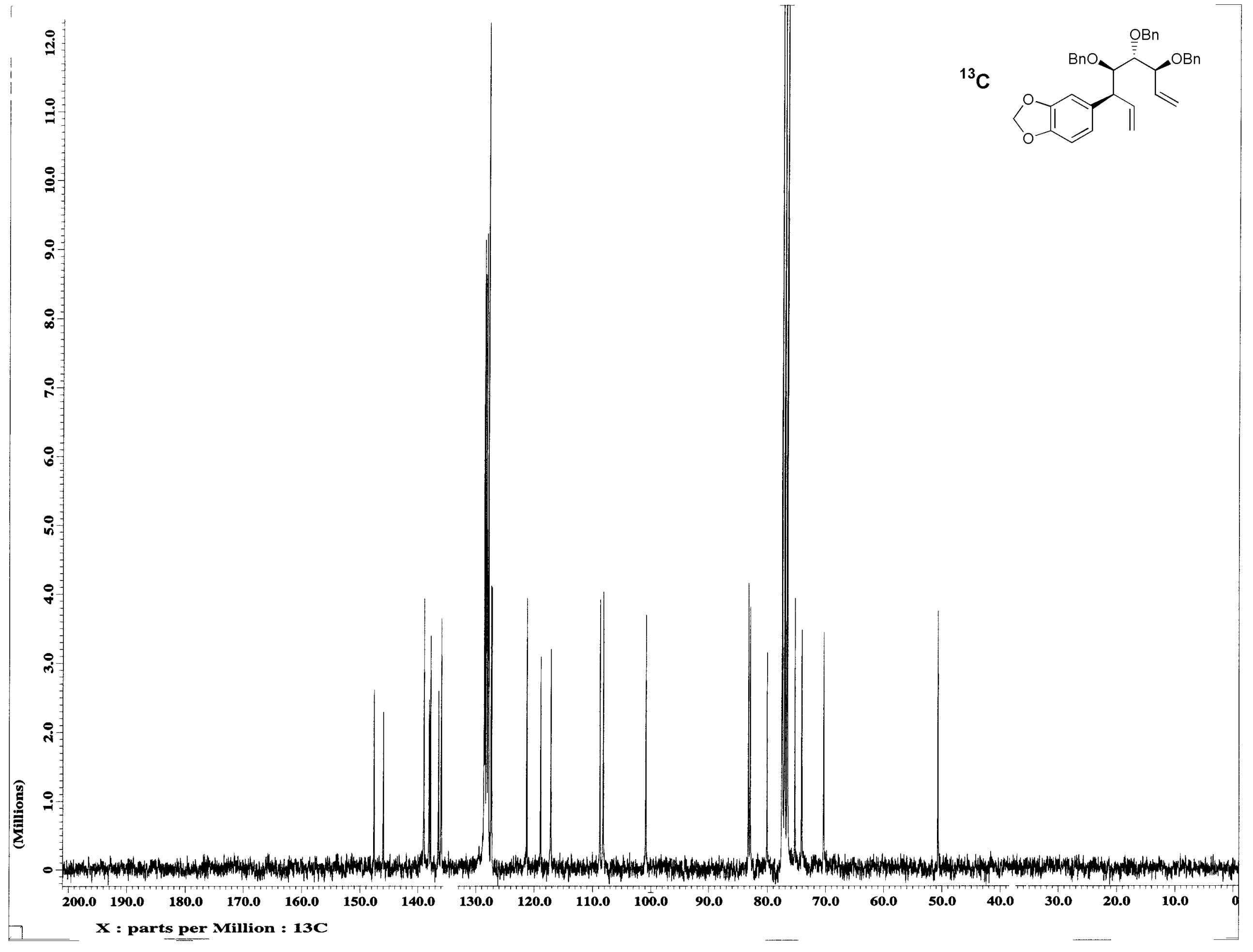




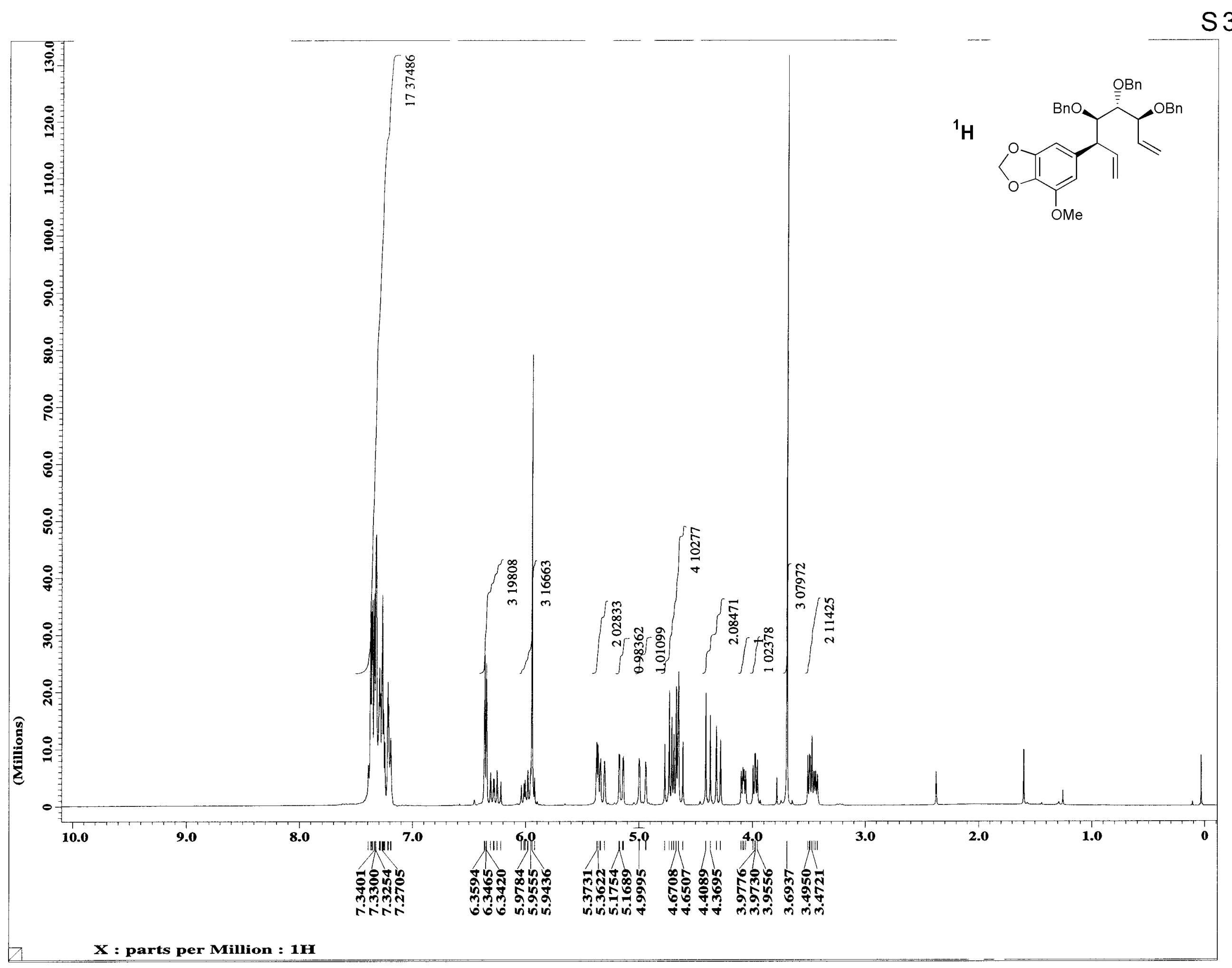




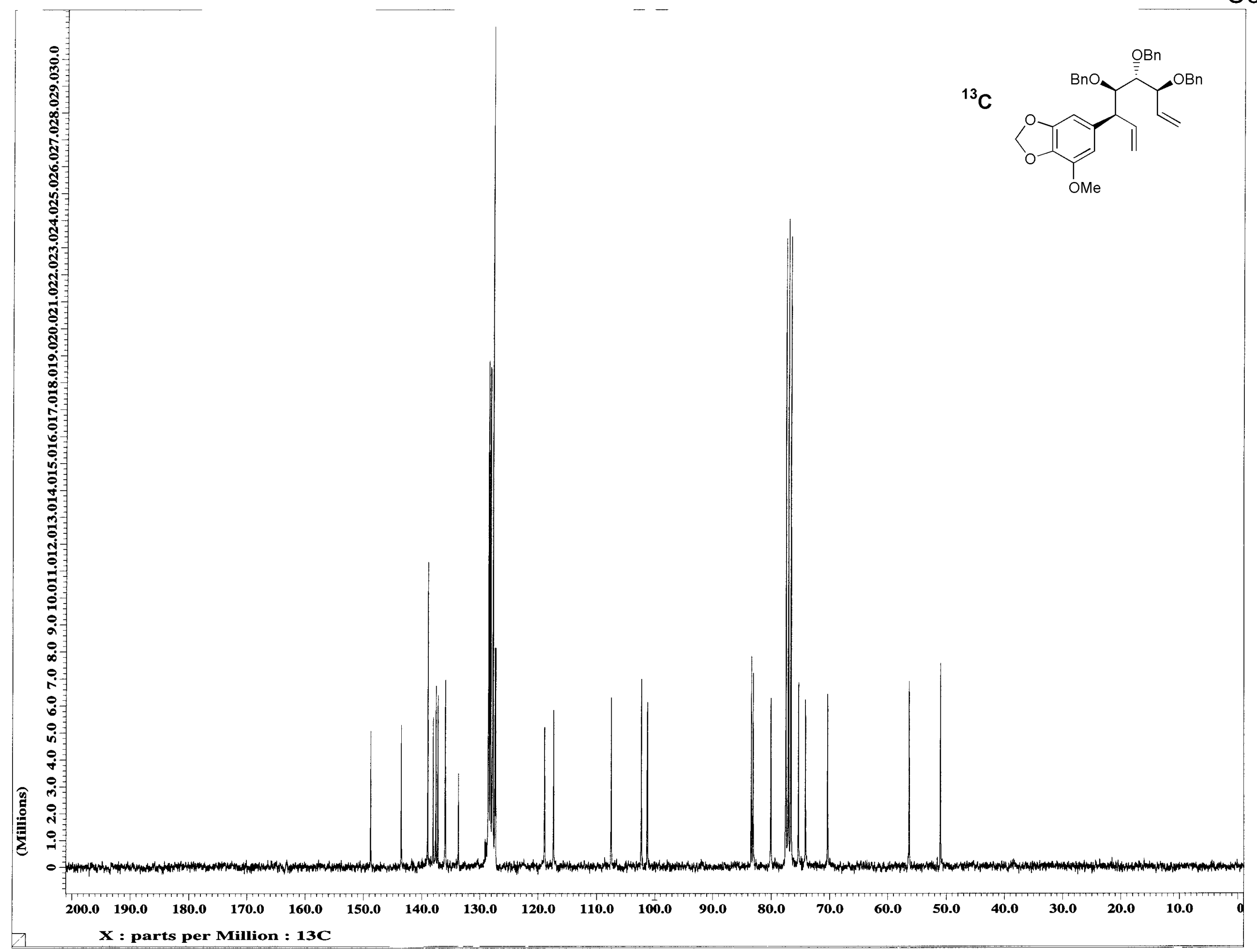




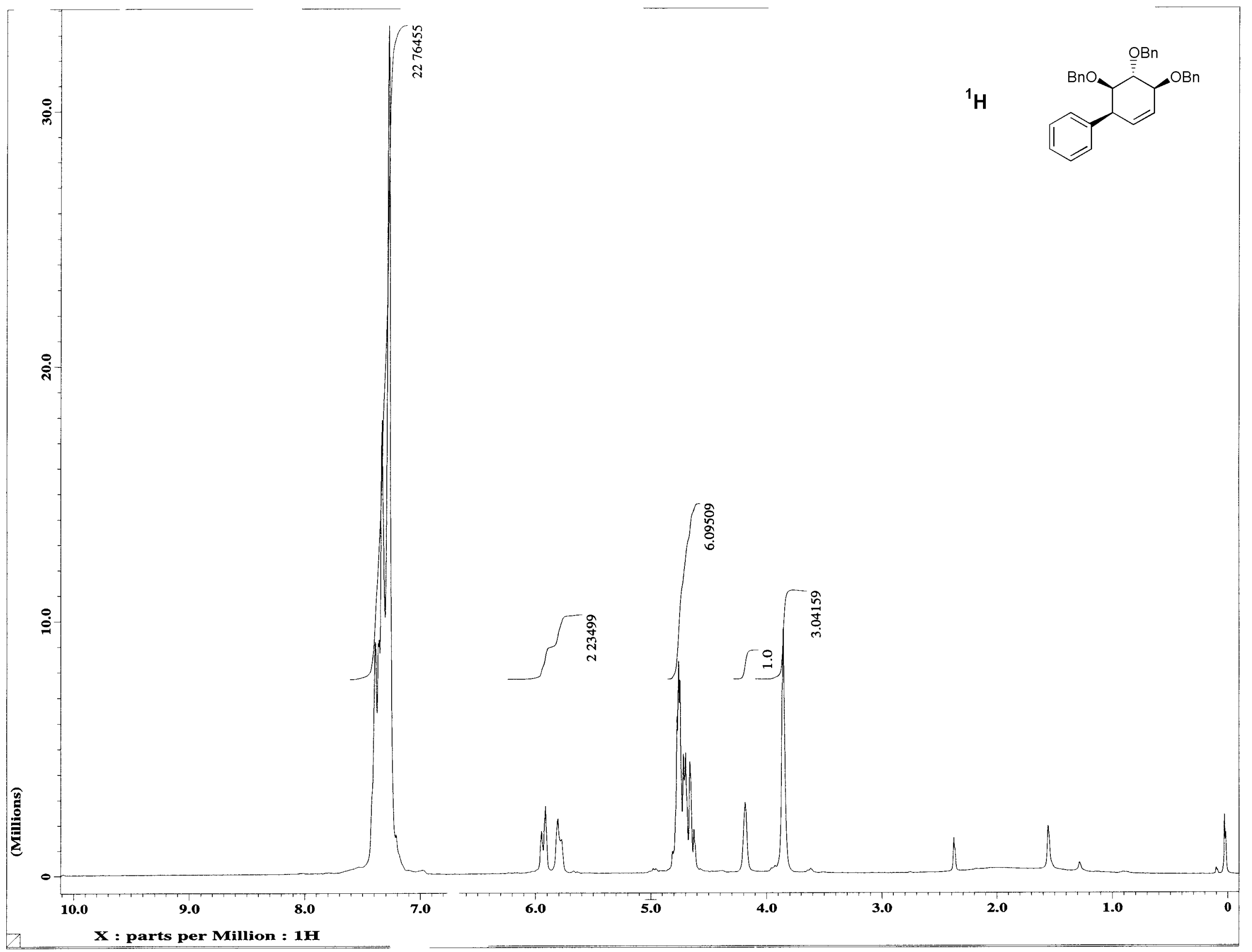




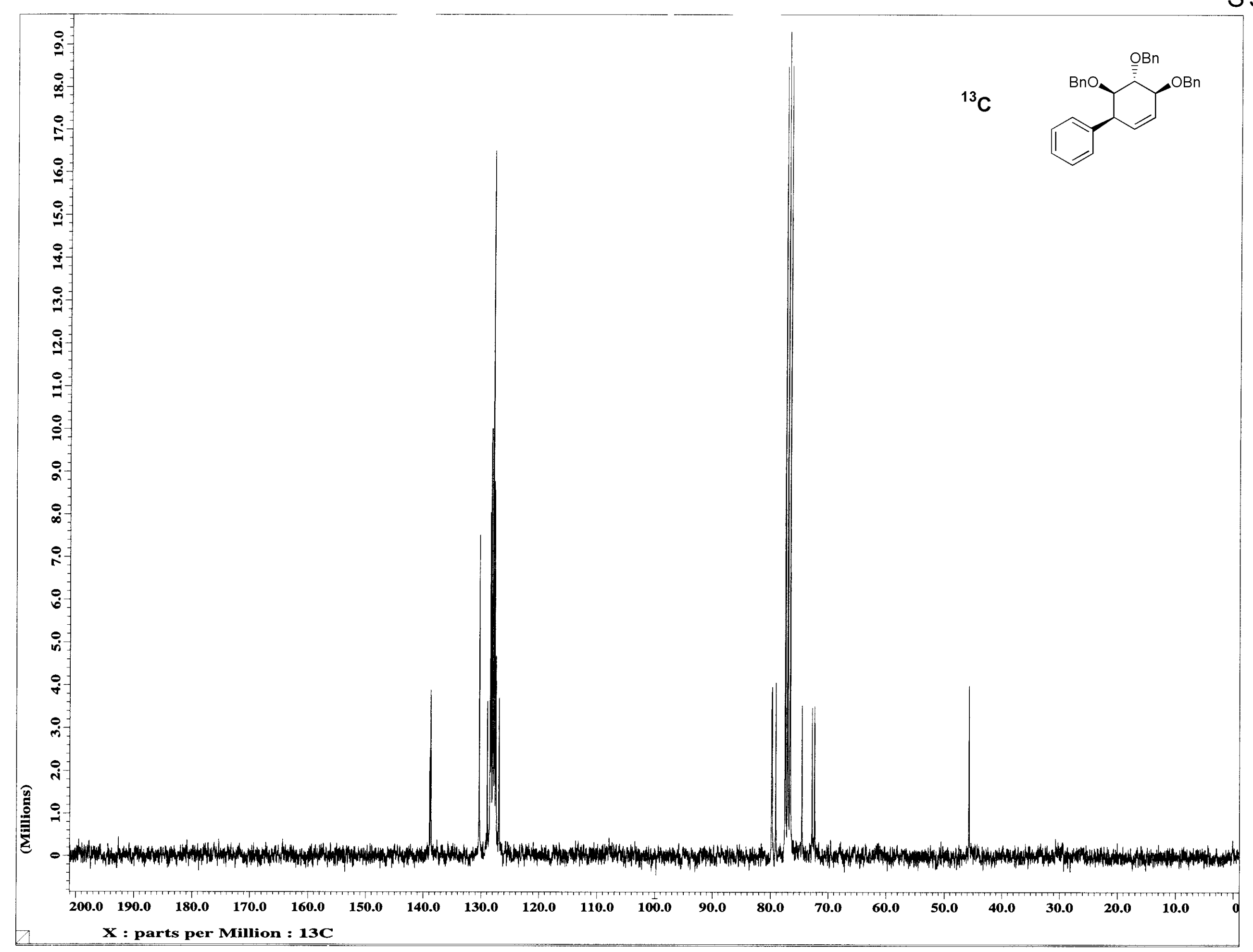




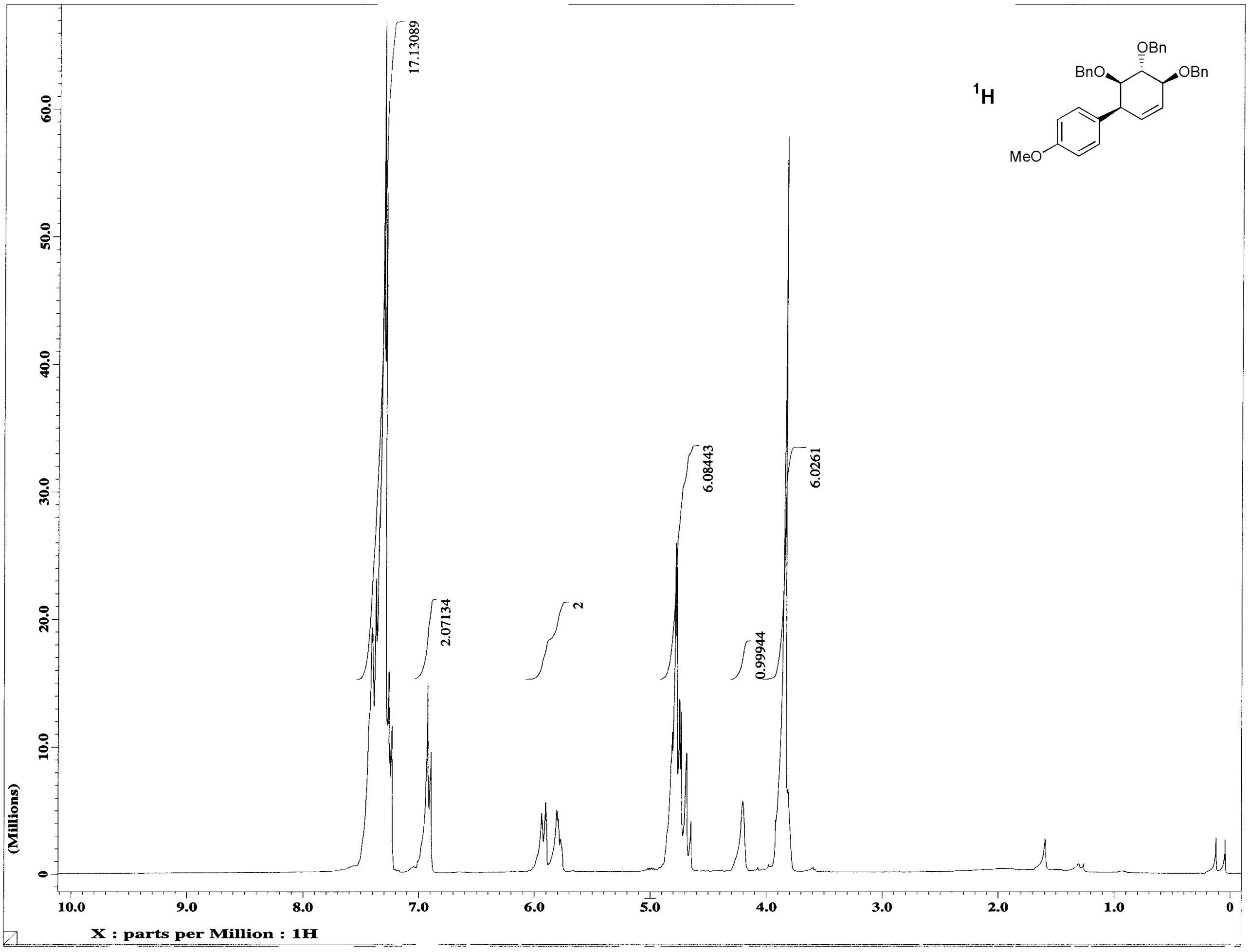




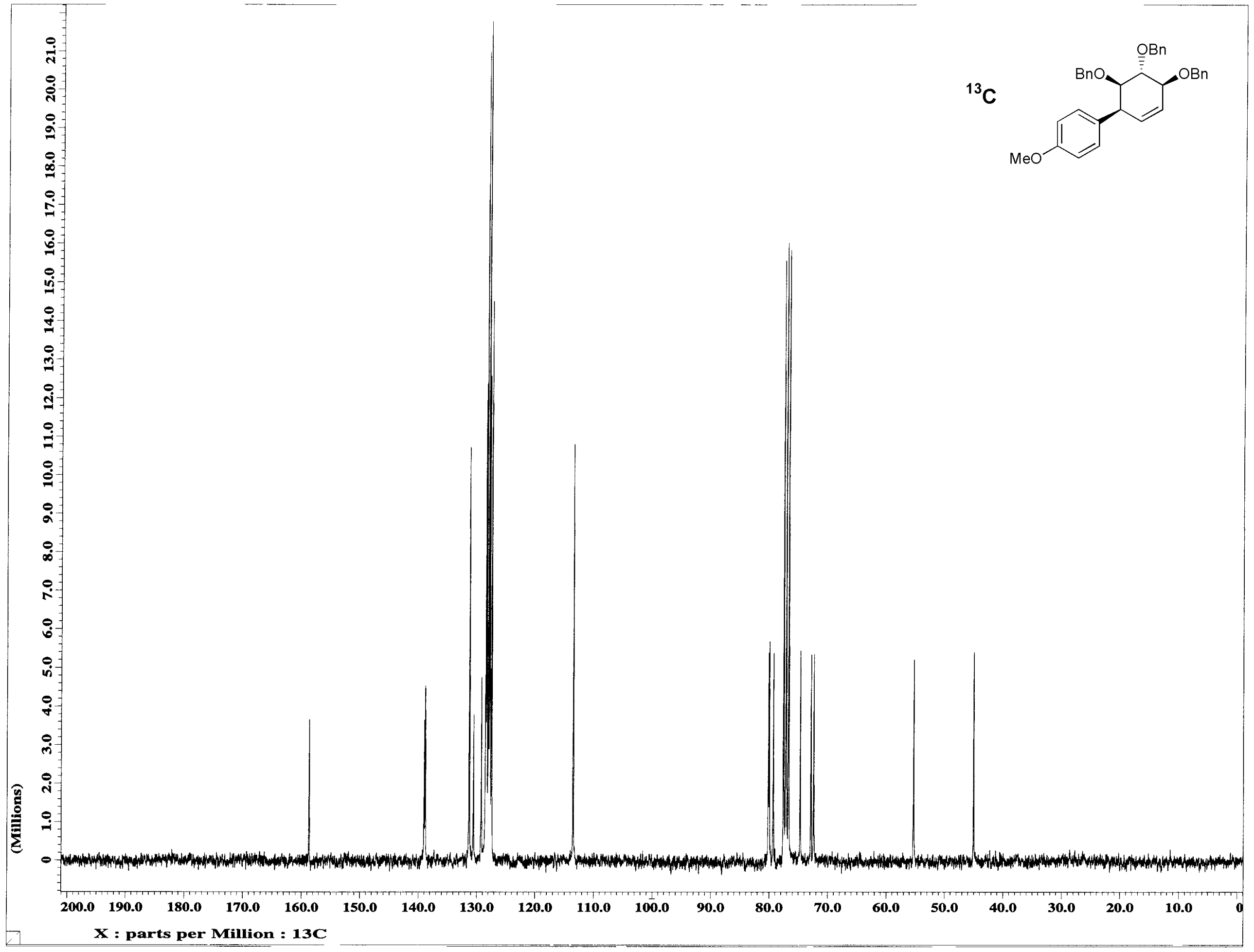




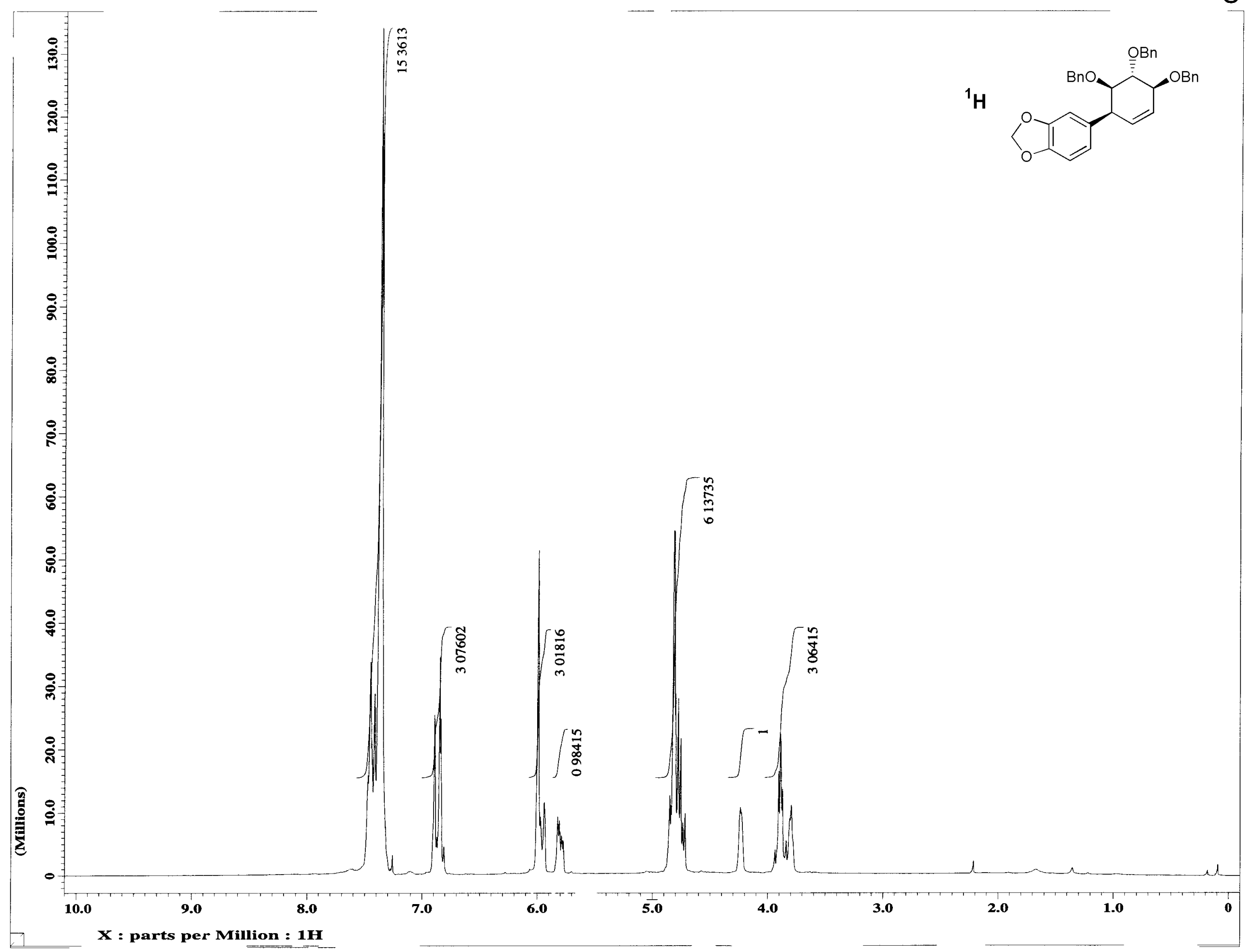




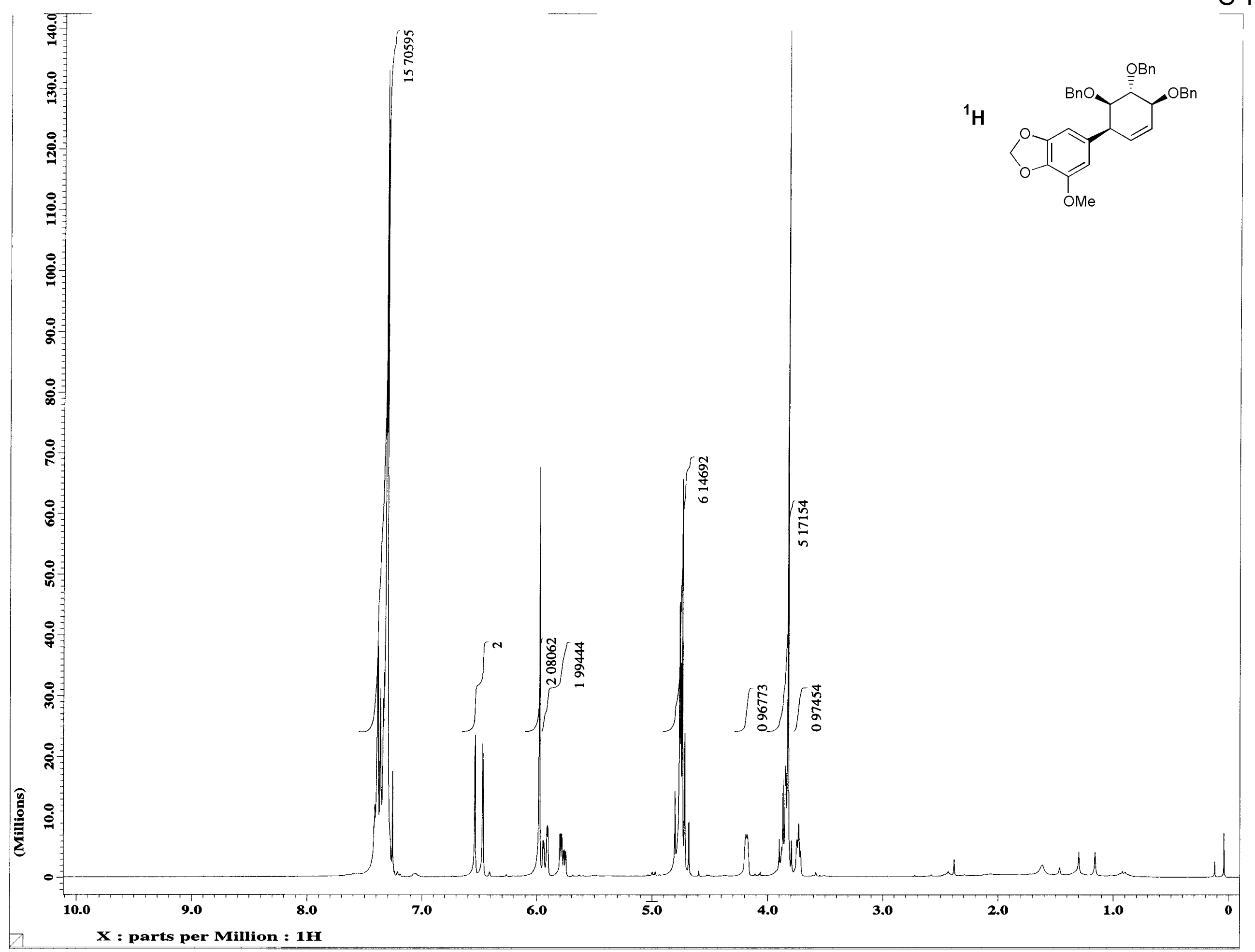




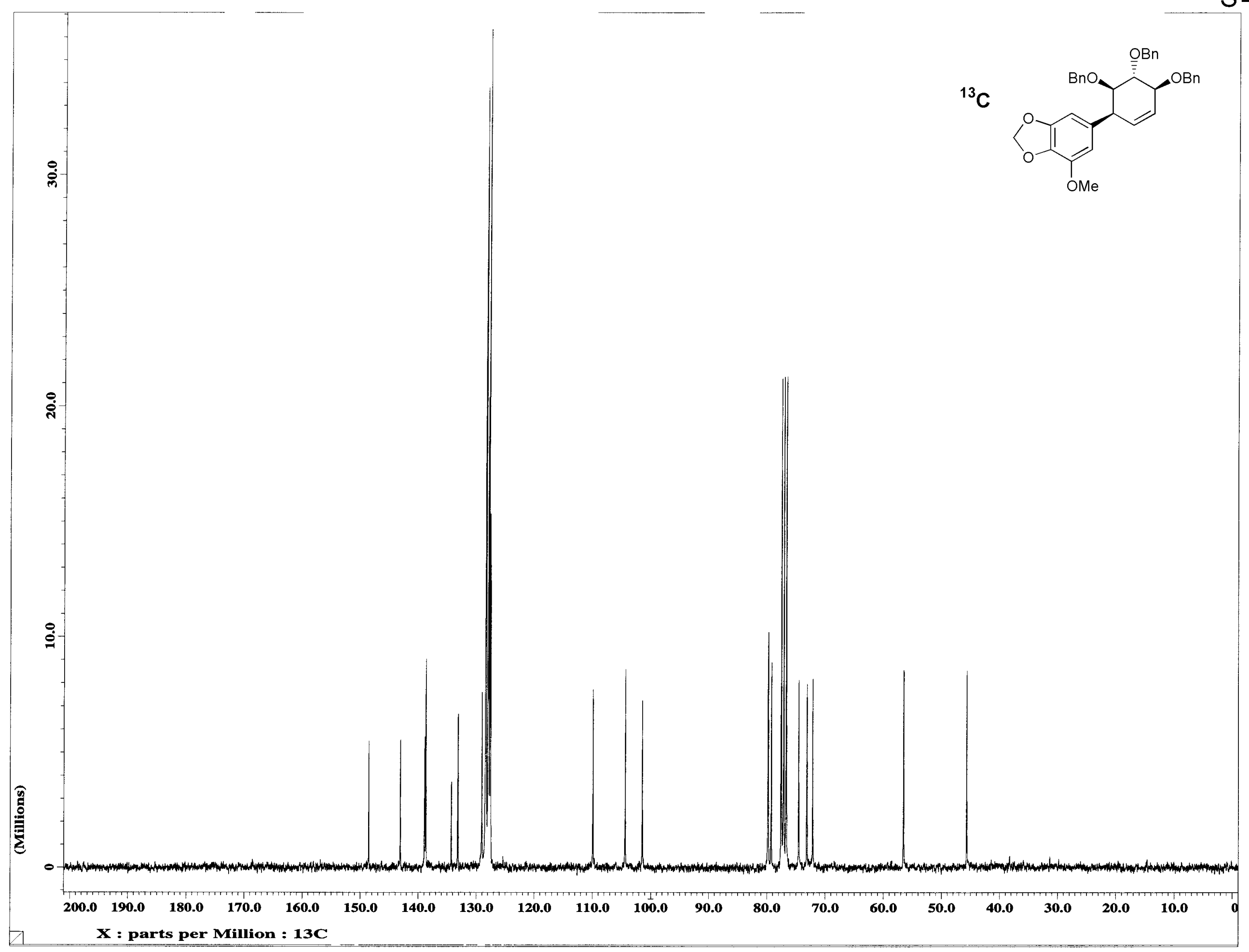




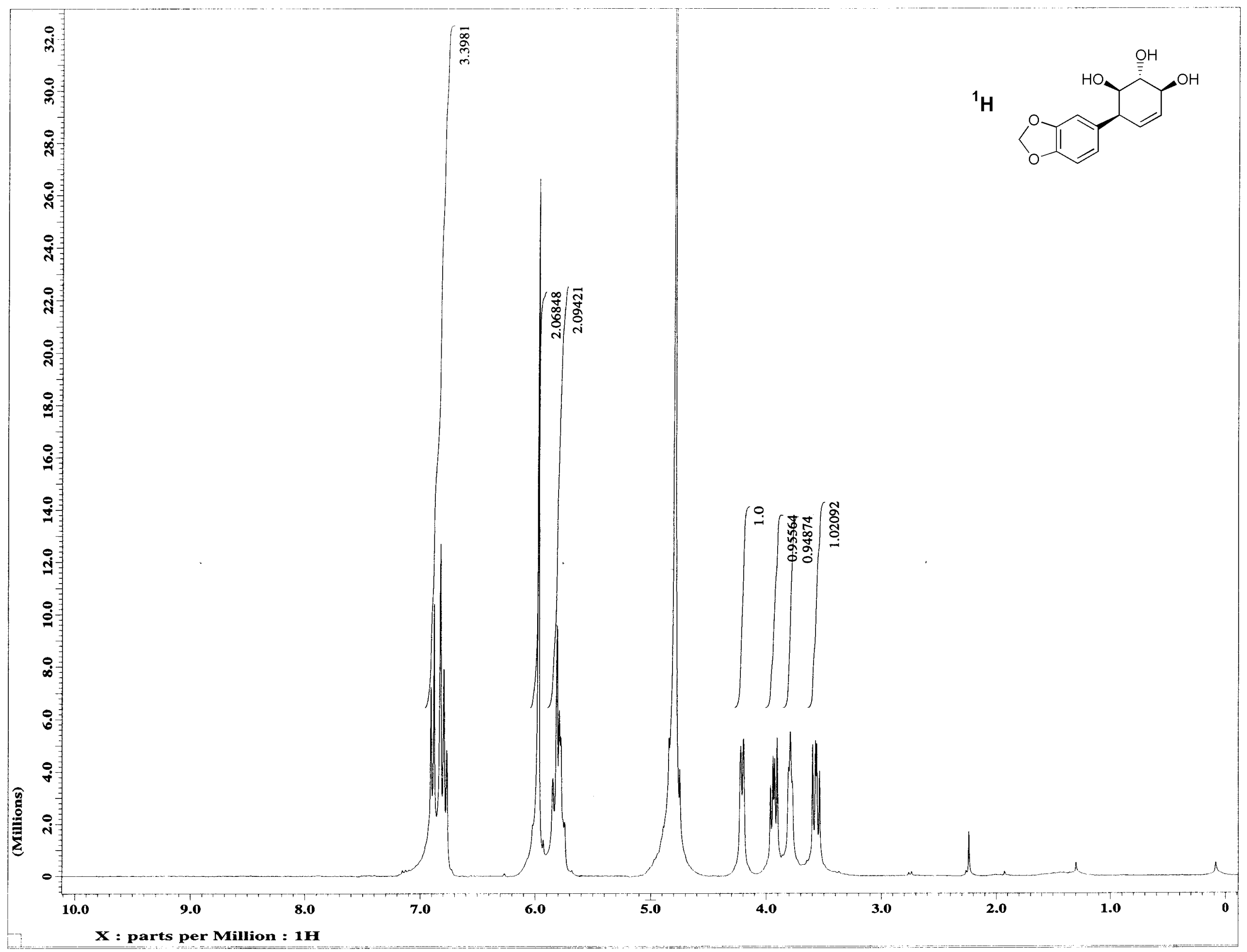




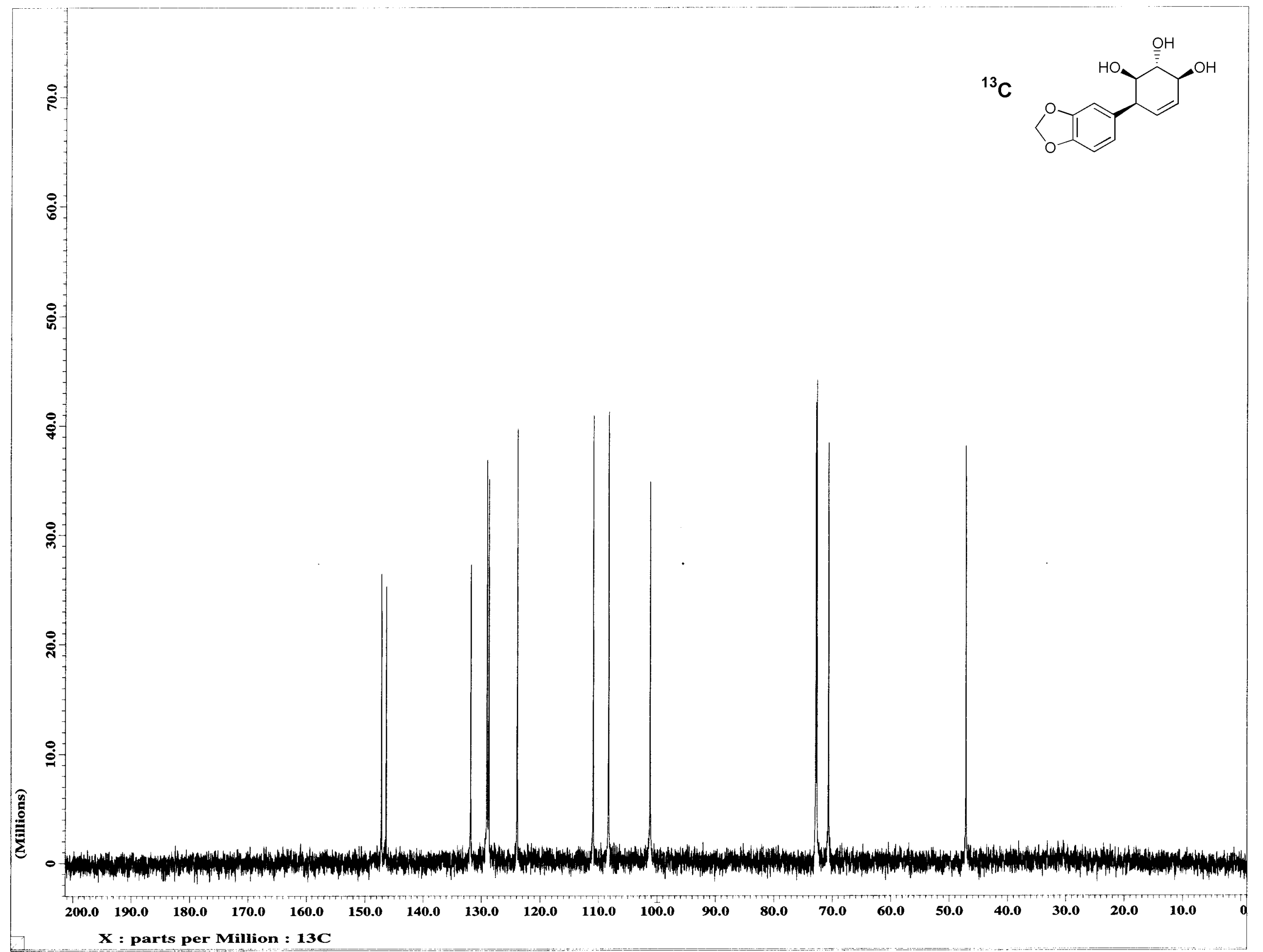




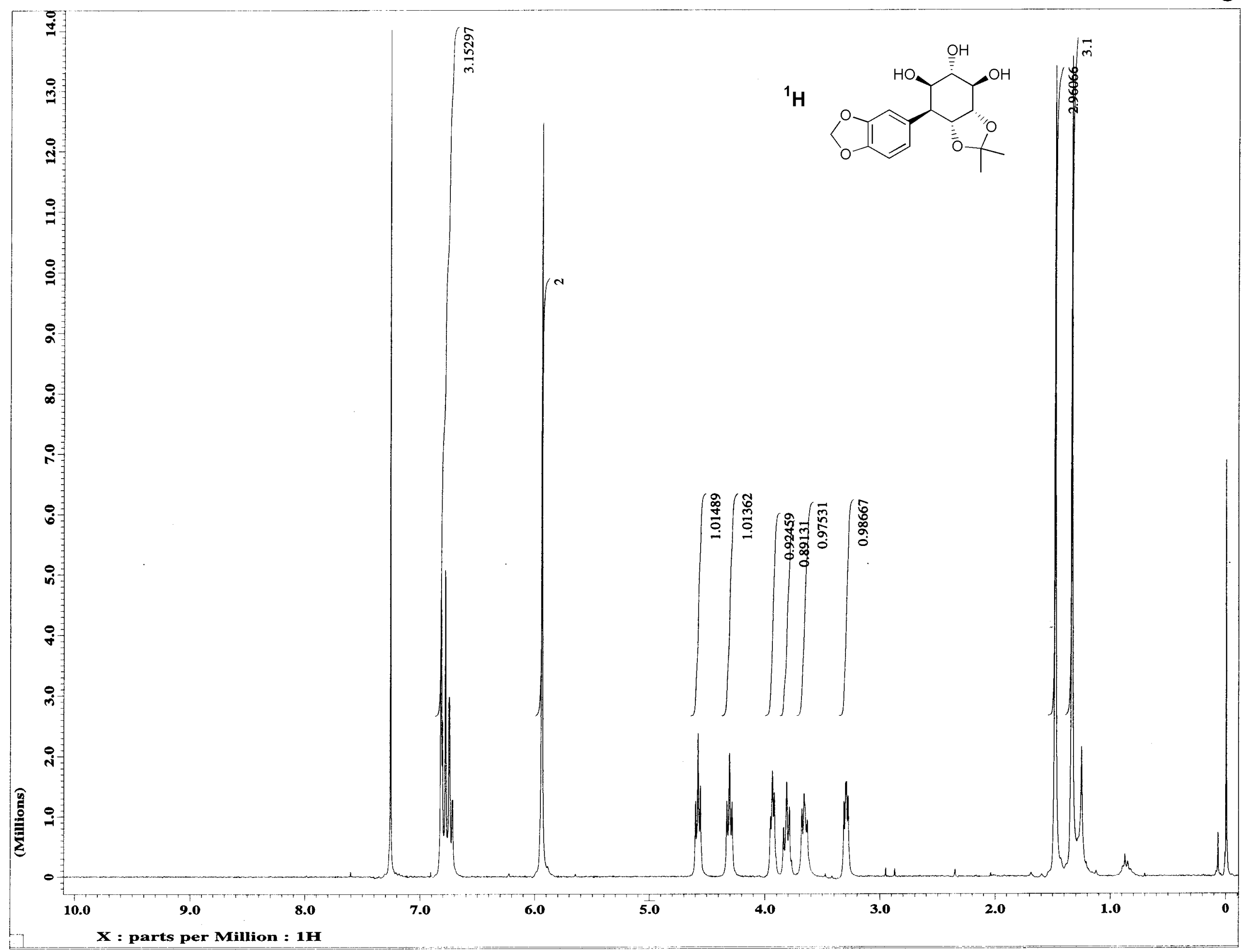




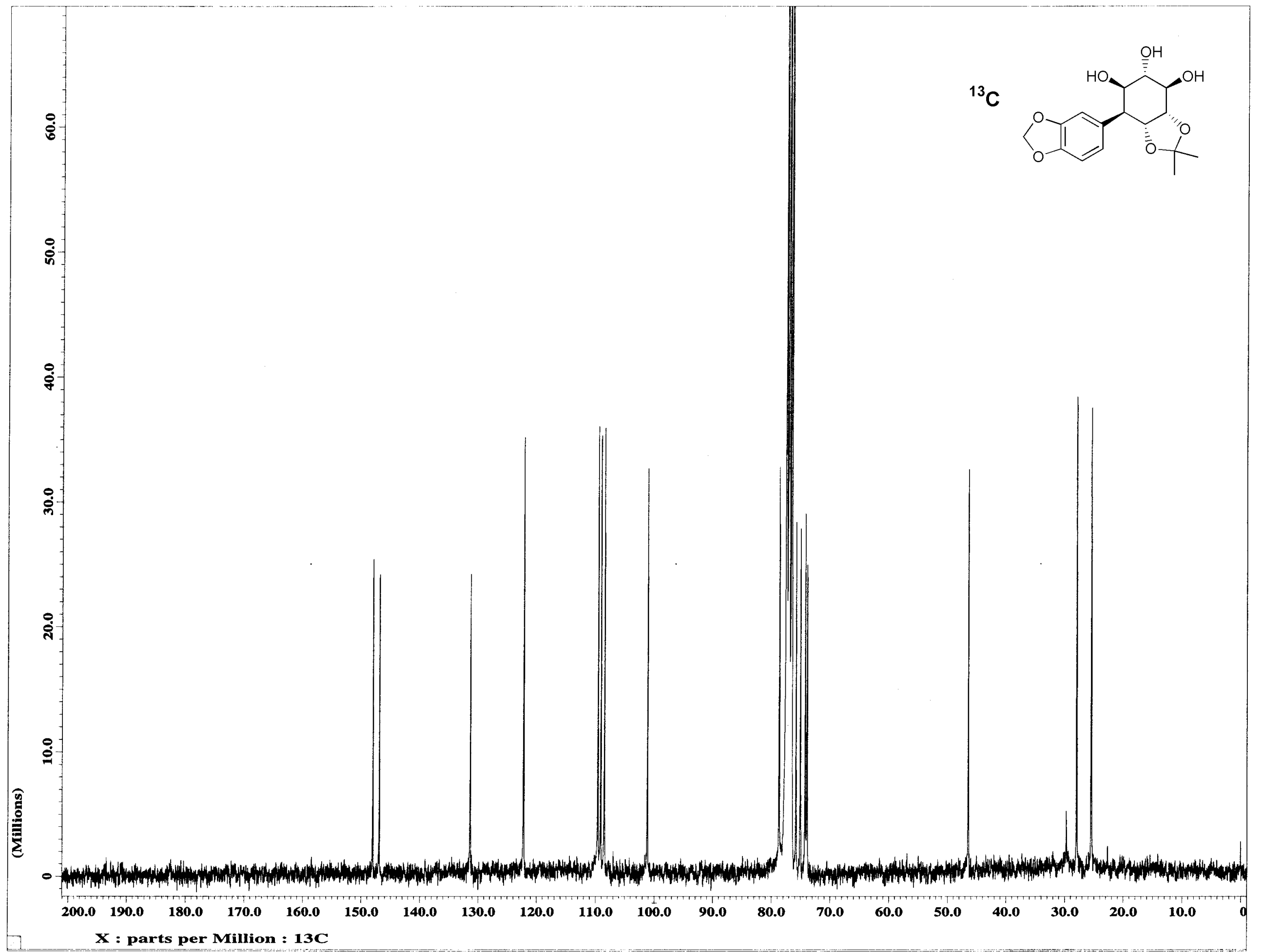




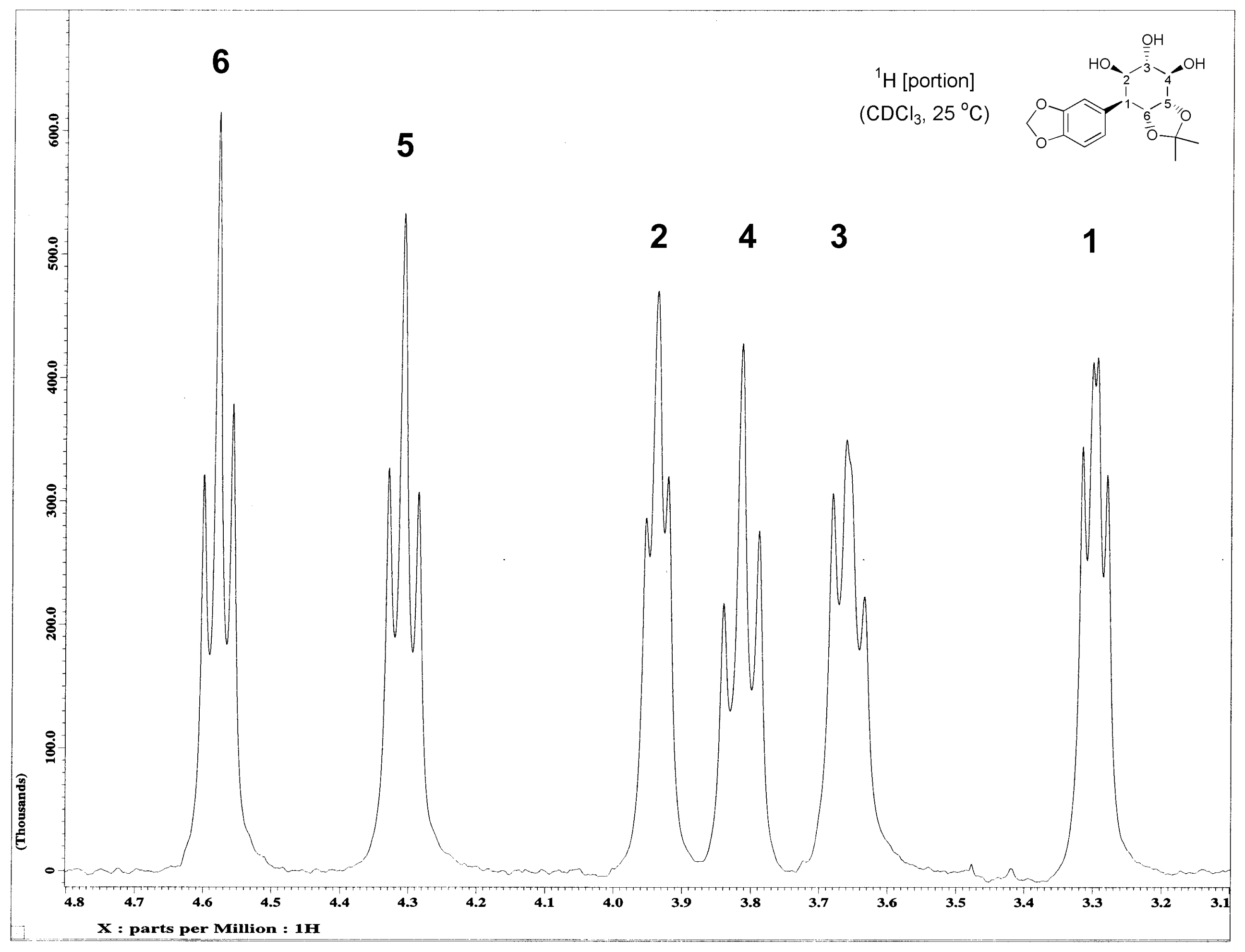




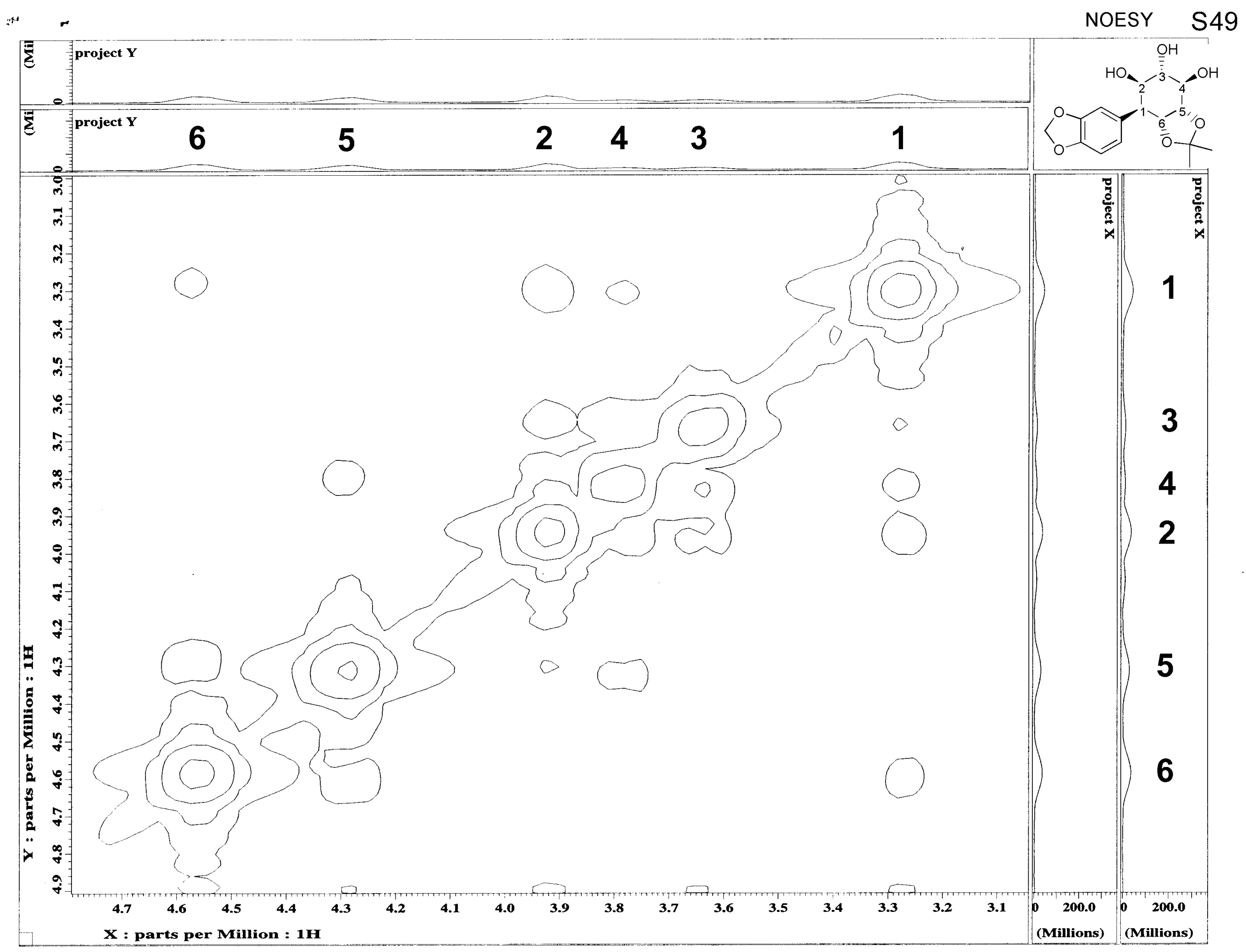


S 50

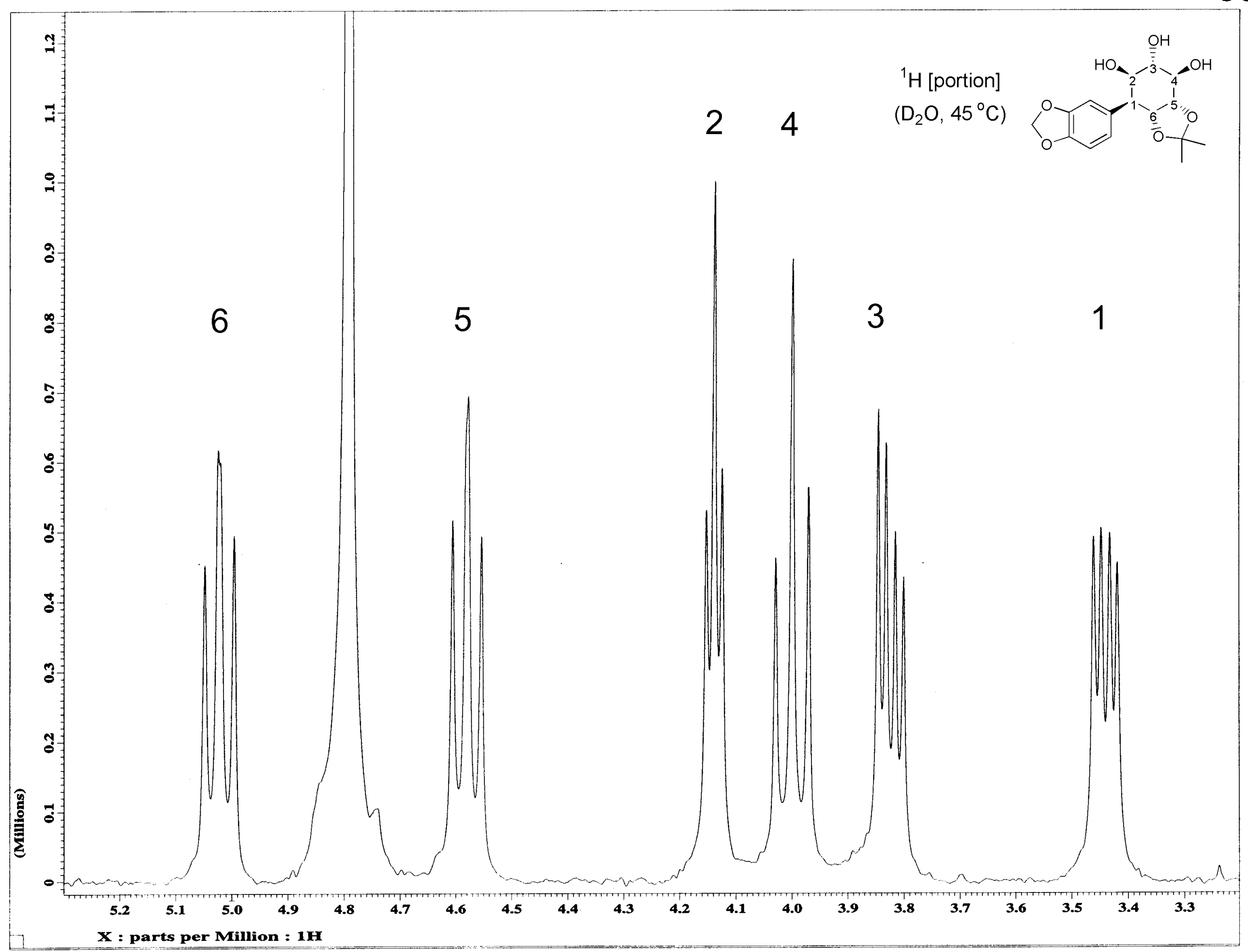




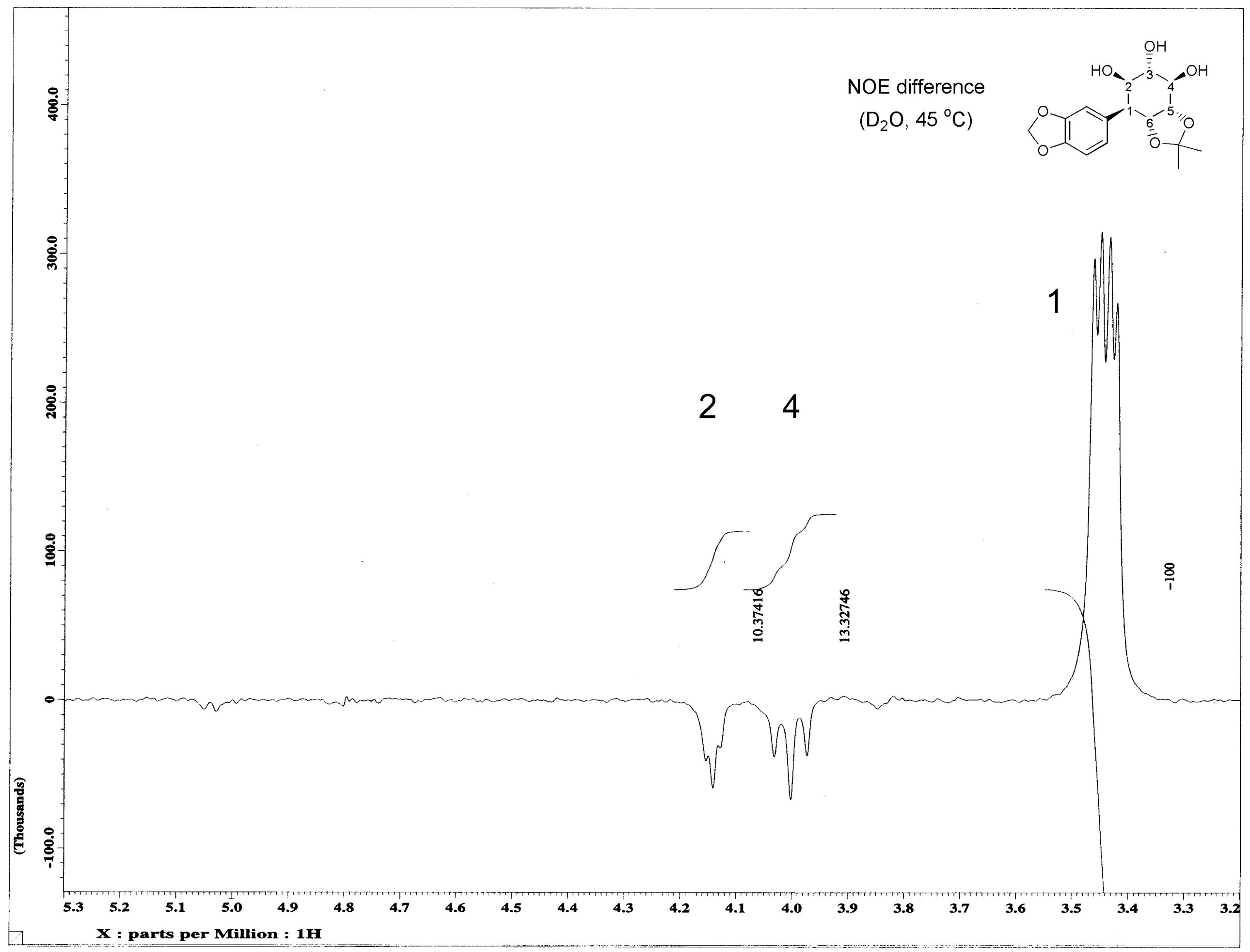

